\title{
The cluster gas mass fraction as a cosmological probe: a revised study ${ }^{\star}$
}

\author{
S. Ettori ${ }^{1,2}$, A. Morandi ${ }^{3,4}$, P. Tozzi ${ }^{5,6}$, I. Balestra ${ }^{7}$, S. Borgani ${ }^{5,6,8}$, P. Rosati ${ }^{9}$, L. Lovisari ${ }^{3,10}$, and F. Terenziani ${ }^{3}$ \\ 1 INAF, Osservatorio Astronomico di Bologna, via Ranzani 1, 40127 Bologna, Italy \\ e-mail: stefano.ettori@oabo.inaf.it \\ 2 INFN, Sezione di Bologna, viale Berti Pichat 6/2, 40127 Bologna, Italy \\ 3 Dipartimento di Astronomia, Università di Bologna, via Ranzani 1, 40127 Bologna, Italy \\ 4 Dark Cosmology Centre, Niels Bohr Institute, University of Copenhagen, Juliane Maries Vej 30, 2100 Copenhagen, Denmark \\ 5 INAF, Osservatorio Astronomico di Trieste, via G.B. Tiepolo 11, 34131 Trieste, Italy \\ 6 INFN, Sezione di Trieste, 34100 Trieste, Italy \\ 7 MPE, Karl-Schwarzschild-Str. 2, 85741 Garching, Germany \\ 8 Dipartimento di Astronomia, Università di Trieste, via Tiepolo 11, 34131 Trieste, Italy \\ 9 ESO, Karl-Schwarzschild-Str. 2, 85748 Garching, Germany \\ ${ }^{10}$ Institut für Astro- und Teilchenphysik, Universität Innsbruck, Technikerstrasse 25, 6020 Innsbruck, Austria
}

Received 29 August 2008 / Accepted 28 March 2009

\section{ABSTRACT}

\begin{abstract}
Context. We present the analysis of the baryonic content of $52 \mathrm{X}$-ray luminous galaxy clusters observed with Chandra in the redshift range $0.3-1.273$.

Aims. Our study aims at resolving the gas mass fraction in these objects to place constraints on the cosmological parameters $\Omega_{\mathrm{m}}, \Omega_{\Lambda}$ and the ratio between the pressure and density of the dark energy, $w$.

Methods. We deproject the X-ray surface brightness profiles to recover the gas mass profiles and fit a single thermal component to the spectrum extracted from a region around the cluster that maximizes the signal-to-noise ratios in the observation. The measured values of the gas temperature are used to evaluate the temperature profile with a given functional form and to estimate the total gravitating mass in combination with the gas density profiles. These measured quantities are then used to statistically estimate the gas fraction and the fraction of mass in stars. By assuming that galaxy clusters are representative of the cosmic baryon budget, the distribution of the cluster baryon fraction in the hottest $\left(T_{\text {gas }}>4 \mathrm{keV}\right)$ systems as a function of redshift is used to constrain the cosmological parameters. We discuss how our constraints are affected by several systematic effects, namely the isothermality, the assumed baryon fraction in stars, the depletion parameter and the sample selection.

Results. By using only the cluster baryon fraction as a proxy for the cosmological parameters, we obtain that $\Omega_{\mathrm{m}}$ is very well constrained at the value of 0.35 with a relative statistical uncertainty of $11 \%(1 \sigma$ level; $w=-1)$ and a further systematic error of about $(-6,+7) \%$. On the other hand, constraints on $\Omega_{\Lambda}$ (without the prior of flat geometry) and $w$ (using the prior of flat geometry) are definitely weaker due to the presence of greater statistical and systematic uncertainties (of the order of 40 per cent on $\Omega_{\Lambda}$ and greater than 50 per cent on $w$ ). If the WMAP 5-year best-fit results are assumed to fix the cosmological parameters, we limit the contributions expected from non-thermal pressure support and ICM clumpiness to be lower than about 10 per cent, also leaving room to accommodate baryons not accounted for either in the X-ray emitting plasma or in stars of the order of 18 per cent of the total cluster baryon budget. This value is lowered to zero for a no-flat Universe with $\Omega_{\Lambda}>0.7$.
\end{abstract}

Key words. galaxies: clusters: general - galaxies: fundamental parameters - intergalactic medium - X-rays: galaxies: clusters cosmology: observations - dark matter

\section{Introduction}

Several tests have been suggested to constrain the geometry and the relative amounts of the matter and energy constituents of the Universe (see Peebles \& Ratra 2003 and references therein). A method that is robust and complementary to others is obtained using the gas mass fraction, $f_{\text {gas }}=M_{\text {gas }} / M_{\text {tot }}$, as inferred from $\mathrm{X}$-ray observations of clusters of galaxies. In this work, we consider two independent methods for our purpose: (i) we compare the relative amount of baryons with respect to the total mass observed in galaxy clusters to the cosmic baryon fraction to provide a direct constraint on $\Omega_{\mathrm{m}}$ (this method was originally adopted by

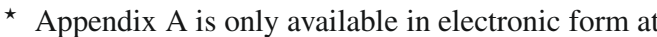
http://www. aanda.org
}

White et al. 1993 to show the limitation of the standard cold dark matter scenario in an Einstein-de Sitter Universe); (ii) we limit the parameters that describe the geometry of the universe by assuming that the gas fraction is constant in time, as first suggested by Sasaki (1996).

Starting from these pioneering works, many studies have followed this approach to constrain the cosmological parameters (White \& Fabian 1995; David et al. 1995; Ettori \& Fabian 1998; Rines et al. 1999; Roussel et al. 2000; Allen et al. 2002; Ettori et al. 2003; Castillo-Morales \& Schindler 2003; Sadat et al. 2005; Allen et al. 2008). We present here a revised and updated version of the work discussed in Ettori et al. (2003, Paper I). The main differences with respect to Paper I are the following: (1) the present dataset contains 60 objects that spans homogeneously the redshift range between 0.06 and 1.27 , whereas in 
the previous analysis 17 objects were selected, 9 at $z \lesssim 0.1$ and 8 at $z>0.7$; (2) several assumptions on, e.g., the star mass fraction and the depletion parameter are tested against different measurements from observations and numerical simulations; (3) a more extensive analysis is performed to assess the systematics that affect our results on the constraints of the cosmological parameters.

The outline of our work is the following. In Sect. 2, we describe the cosmological framework that allows us to formulate the cosmological dependence of the cluster gas mass fraction. In Sect. 3, the dataset is presented and the physical quantities under examination are computed. The method based on using the gas mass fraction as a cosmological probe is discussed in Sect. 4. The results on the cosmological parameters are shown in Sect. 5, along with a discussion on their robustness against systematic effects. The summary of our findings and the conclusions are drawn in Sect. 6. Throughout this work, if not otherwise stated, we plot and tabulate values, with errors quoted at the 68.3 per cent $(1 \sigma)$ level of confidence, that are estimated by assuming $H_{0}=70 h_{70}^{-1} \mathrm{~km} \mathrm{~s}^{-1} \mathrm{Mpc}^{-1}$ and $\Omega_{\mathrm{m}}=1-\Omega_{\Lambda}=0.3$.

\section{The cosmological framework}

We refer to $\Omega_{\mathrm{b}}$ as the baryon matter density, to $\Omega_{\mathrm{m}}$ as the total matter density (i.e. $\Omega_{\mathrm{m}}=\Omega_{\mathrm{b}}+\Omega_{\mathrm{c}}$, where $\Omega_{\mathrm{c}}$ is the cold dark matter component), to $\Omega_{\Lambda}$ as the dark energy density, that we consider both in its static and homogeneous form as a cosmological constant and with an equation of state varying with cosmic time as "quintessence" (e.g. Turner \& White 1997; Caldwell et al. 1998). All these densities are expressed in units of the critical density, $\rho_{\mathrm{c}}=3 H_{0}^{2} /(8 \pi G)$, where $H_{0}$ is Hubble's constant and $G$ is the gravitational constant. In our computation, we neglect (i) the energy associated with the cosmic radiation, $\Omega_{\mathrm{r}} \approx 4.16 \times 10^{-5}\left(T_{\mathrm{CMB}} / 2.726 \mathrm{~K}\right)^{4}$; and (ii) any possible contributions from light neutrinos, $\Omega_{v} h_{70}^{2}=\sum m_{v} / 45.5 \mathrm{eV}$, that is expected to be less than 0.01 for a total mass in neutrinos, $\sum m_{v}$, lower than $0.62 \mathrm{eV}$ (see, e.g., Hannestad \& Raffelt 2006). Therefore, we adopt the Einstein equation in the form $\Omega_{\mathrm{m}}+\Omega_{\Lambda}+\Omega_{\mathrm{k}}=1$, where $\Omega_{\mathrm{k}}$ accounts for the curvature of space. From, e.g., Carroll et al. (1992, cf. Eq. (25)), we can then write the angular diameter distance as

$$
\begin{aligned}
d_{\text {ang }} & =\frac{d_{\text {lum }}}{(1+z)^{2}}=\frac{c}{H_{0}(1+z)} \frac{S(\omega)}{\left|\Omega_{\mathrm{k}}\right|^{1 / 2}}, \\
\omega & =\left|\Omega_{\mathrm{k}}\right|^{1 / 2} \int_{0}^{z} \frac{\mathrm{d} \zeta}{E(\zeta)},
\end{aligned}
$$

where $d_{\text {lum }}$ is the luminosity distance, $S(\omega)$ is $\sinh (\omega), \omega, \sin (\omega)$ for $\Omega_{\mathrm{k}}$ greater than, equal to and less than 0 , respectively. In addition, we define

$$
\begin{aligned}
& E(z)=\left[\Omega_{\mathrm{m}}(1+z)^{3}+\Omega_{\mathrm{k}}(1+z)^{2}+\Omega_{\Lambda} \lambda(z)\right]^{1 / 2} \\
& \lambda(z)=\exp \left(3 \int_{0}^{z} \frac{1+w(z)}{1+z} \mathrm{~d} z\right) .
\end{aligned}
$$

The above equations (i) do not include the evolution with redshift of the radiation component, $\Omega_{\mathrm{r}}(1+z)^{4}$, that at $z \approx 1$ is about $1.4 \times 10^{-3}$ and therefore negligible in the overall budget; (ii) consider the dependence upon the ratio $w$ between the pressure and the energy density in the equation of state of the dark energy component (Caldwell et al. 1998; Wang \& Steinhardt 1998). Hereafter we consider both a pressure-to-density ratio $w$ constant in time that implies $\lambda(z)=(1+z)^{3+3 w}$ and a simple parameterization of its evolution with redshift (e.g. Rapetti et al. 2005; Firmani et al. 2005)

$w(z)=w_{0}+w_{1} \frac{z}{1+z}$

In particular, the case of a cosmological constant requires $w=$ -1 .

\section{The dataset}

We consider the sample of 52 galaxy clusters at $z>0.3$ presented in Balestra et al. (2007; see Table 1). The preparation, reduction and analysis of this dataset is described below.

As local measurements, we consider the sample of 8 objects at a redshift between 0.06 and 0.23 with a gas temperature higher than $4 \mathrm{keV}$ described in Vikhlinin et al. (2006; see Table 2). We use the best-fit results of the gas temperature and density profiles obtained by adopting the same functional form that is applied in the present analysis to reproduce the ICM properties of the high$z$ sample. The gas and total mass profiles are then recovered for both the local and the $z>0.3$ sample with the same method described at the end of this section.

The histogram of the redshift distribution of the two samples is shown in Fig. 1.

The only criterion adopted for the selection of these objects is the availability of good exposures with Chandra of hot, massive, relaxed galaxy clusters over a significant redshift range. No selection effect is expected to occur in the application of the gas mass fraction method once the clusters are selected to ensure: (i) the use of the hydrostatic equilibrium equation to recover the total mass (round, relaxed objects are required for that); and (ii) a negligible contribution from non-gravitational energy in the region of interest to allow the use of cross-calibration with numerical simulations, such as the estimate of the depletion parameter (the selection of hot, massive systems dominated energetically by gravitational collapse satisfies this condition).

We have considered only Chandra exposures of the clusters listed in Table 1. Data reduction is performed using the CIAO 3.2 software package with a recent version of the Calibration Database (CALDB 3.0.0) including the correction for the degraded effective area of ACIS-I chips due to material accumulated on the ACIS optical blocking filter at the epoch of the observation. We also apply the time-dependent gain correction ${ }^{1}$, which is necessary to adjust the "effective gains", which drifts with time due to an increasing charge transfer inefficiency (CTI). The detailed procedure of data reduction is described in Balestra et al. (2007).

A single thermal component (XSPEC model mekal; Arnaud 1996) absorbed by the Galactic column density is fitted to the spectrum extracted from a circular region with radius $R_{\text {spec }}$ (see Table 1) around the cluster chosen to maximize the signal-tonoise ratio once the contaminating point sources are masked. The only free parameters in the spectral fit are the temperature, the normalization and the metallicity with respect to the abundance table from Grevesse \& Sauval (1998) here assumed as the reference. These temperature measurements are on average 3 per cent higher than the values obtained by assuming the standard metallicity table of Anders \& Grevesse (1989).

We assume that galaxy clusters are spherically symmetric gravitationally bound systems. We deproject the X-ray surface brightness to obtain the electron density profile, $n_{\mathrm{e}}(r)$, by correcting the emissivity by the contribution from the outer shells

1 http://asc.harvard.edu/ciao/threads/acistimegain/ 
Table 1. Properties of the $z>0.3$ cluster sample described in Balestra et al. (2007).

\begin{tabular}{|c|c|c|c|c|c|c|c|c|c|}
\hline Cluster & $z$ & $\begin{array}{l}k T_{\text {gas }} \\
\mathrm{keV}\end{array}$ & $\left(a_{0}, a_{1}, a_{2} / 10^{-3}, a_{3}, a_{4}, a_{6}\right)$ & $\begin{array}{c}R_{\text {spat }} \\
\text { kpc }\end{array}$ & $\begin{array}{c}R_{\mathrm{spec}} \\
\mathrm{kpc}\end{array}$ & $\begin{array}{l}R_{500} \\
\mathrm{kpc}\end{array}$ & $\begin{array}{c}f_{\text {gas }} \\
\left(<R_{500}\right)\end{array}$ & $\begin{array}{c}\hat{R}_{500} \\
\mathrm{kpc}\end{array}$ & $\begin{array}{c}\hat{f}_{\text {gas }} \\
\left(<R_{500}\right)\end{array}$ \\
\hline MS1008.1 - 1224 & 0.306 & $6.90 \pm 0.35$ & $(0.928,0.028,3.9,0.39,1.13,0.44)$ & 688 & 490 & $1176 \pm 116$ & $0.135 \pm 0.045$ & $1303 \pm 179$ & $0.112 \pm 0.044$ \\
\hline MS2137.3 - 2353 & .313 & $5.05 \pm 0.11$ & $(0.028,0.204,93.3,0.37,0.53,0.47)$ & 698 & 362 & $940 \pm 26$ & $0.152 \pm 0.016$ & $997 \pm 35$ & $0.135 \pm 0.016$ \\
\hline A1995 & 320 & $8.61 \pm 0.35$ & $(0.142,0.290,7.3,0.32,0.30,1.56)$ & 855 & 479 & $1317 \pm 46$ & $0.091 \pm 0.011$ & $1429 \pm 60$ & $0.076 \pm 0.010$ \\
\hline MACS-J0308.9 + 2645 & 324 & $12.73 \pm 1.02$ & $(0.104,0.230,11.2,0.30,0.30,0.96)$ & 1039 & 577 & $1423 \pm 68$ & $0.131 \pm 0.020$ & $1491 \pm 77$ & $0.120 \pm 0.017$ \\
\hline ZW1358.1 + 6245 & 0.328 & $6.78 \pm 0.28$ & $(0.006,0.400,143.5,0.00,0.35,1.40)$ & 869 & 419 & & & $1256 \pm 100$ & $0.090 \pm 0.019$ \\
\hline MACS-J0404.6 + 1109 & .355 & $7.38 \pm 0.90$ & $(0.105,0.125,4.6,0.00,0.30,0.19)$ & 1398 & 782 & $887 \pm 63$ & $0.205 \pm 0.039$ & $850 \pm 68$ & $0.215 \pm 0.038$ \\
\hline RXJ0027.6 + 2616 & .367 & $8.76 \pm 1.69$ & $(0.676,0.163,2.0,0.35,0.89,0.62)$ & 642 & 550 & $1299 \pm 269$ & $0.067 \pm 0.054$ & $1421 \pm 351$ & $0.055 \pm 0.048$ \\
\hline MACS-J1720.2 + 3536 & .391 & $6.46 \pm 0.33$ & $(0.010,0.533,161.4,0.00,0.40,2.03)$ & 974 & 546 & $1200 \pm 104$ & $0.103 \pm 0.034$ & $1345 \pm 143$ & $0.078 \pm 0.028$ \\
\hline ZW0024.0 + 1652 & 0.395 & $4.32 \pm 0.32$ & $(0.375,0.010,13.2,0.17,0.66,0.60)$ & 672 & 341 & $879 \pm 100$ & $0.098 \pm 0.035$ & $957 \pm 144$ & $0.083 \pm 0.031$ \\
\hline$V 1416+4446$ & 400 & $3.43 \pm 0.20$ & $(0.008,0.544,72.5,0.00,0.36,2.19)$ & 677 & 397 & $797 \pm 100$ & $0.135 \pm 0.058$ & $894 \pm 148$ & $0.110 \pm 0.058$ \\
\hline MACS-J0159.8 - 0849 & 0.405 & $9.43 \pm 0.61$ & $(0.011,0.302,167.0,0.09,0.40,0.86)$ & 994 & 639 & $1214 \pm 62$ & $0.139 \pm 0.025$ & $1286 \pm 81$ & $0.125 \pm 0.022$ \\
\hline MACS-J2228.5 + 2036 & 0.412 & $8.25 \pm 0.59$ & $(0.240,0.079,6.4,0.42,0.48,0.23)$ & 1211 & 753 & $1052 \pm 61$ & $0.217 \pm 0.033$ & $1091 \pm 82$ & $0.205 \pm 0.032$ \\
\hline MS0302.7 + 1658 & 0.424 & $4.78 \pm 0.60$ & & 369 & 328 & $1041 \pm 92$ & & $1167 \pm 104$ & $0.041 \pm 0$. \\
\hline MS1621.5 + 2640 & 0.426 & $7.22 \pm 0.75$ & $(0.248,0.212,3.9,0.12,0.30,0.96)$ & 1003 & 658 & $977 \pm 79$ & $0.153 \pm 0.040$ & $1006 \pm 106$ & $0.146 \pm 0.039$ \\
\hline MACS-J0417.5 - 1154 & .440 & $10.84 \pm 0.98$ & $(0.015,1.000,137.1,0.00,0.40,1.58)$ & 1259 & 785 & $1340 \pm 105$ & $0.201 \pm 0.043$ & $1465 \pm 141$ & $0.171 \pm 0.036$ \\
\hline MACS-J1206.2 - 0847 & 0.440 & $11.98 \pm 0.85$ & $(0.012,0.316,82.9,0.00,0.30,1.11)$ & 1259 & 785 & $1336 \pm 75$ & $0.161 \pm 0.0$ & $1416 \pm 95$ & $0.144 \pm 0.025$ \\
\hline CL1641 + 4001 & 0.464 & $4.74 \pm 0.54$ & $5,1.7,0.44,0.30,1.34)$ & 341 & 287 & $848 \pm 139$ & $0.079 \pm 0.059$ & $902 \pm 189$ & $0.070 \pm 0.055$ \\
\hline MACS-J1621.4 + 3810 & 465 & $6.62 \pm 0.74$ & $0.30,1.14)$ & 893 & 458 & & & $1037 \pm 146$ & \\
\hline $4.3+4309$ & .487 & $9.69 \pm 2.82$ & $(0.0$ & 765 & 50 & 89 & 72 & $873 \pm 223$ & 69 \\
\hline MACS-J1311.0 - 0311 & .492 & $8.17 \pm 0.86$ & & 631 & 478 & $1055 \pm 72$ & & $1112 \pm 85$ & $0.096 \pm 0.023$ \\
\hline $\mathrm{V} 1525+0958$ & 0.516 & $5.54 \pm 0.40$ & & 834 & 490 & $901 \pm 113$ & & $1002 \pm 168$ & $0.115 \pm 0.048$ \\
\hline MS0451.6 - 0305 & .540 & $9.11 \pm 0.45$ & $2,14.4,0.02,0.82,0.71)$ & 1167 & 625 & $1292 \pm 86$ & $0.129 \pm 0.0$ & $1446 \pm 124$ & $0.099 \pm 0.020$ \\
\hline & & & & 1408 & & $7 \pm 54$ & & $1279 \pm 78$ & \\
\hline$-J 1149.5+2223$ & 544 & $13.10 \pm 1.12$ & & 1147 & 944 & $6 \pm 150$ & 31 & $1734 \pm 201$ & $0.079 \pm 0.025$ \\
\hline MACS-J1423.8 + 2404 & 545 & $7.55 \pm 0.59$ & $2,2.50)$ & 805 & 504 & $6 \pm 138$ & & $1343 \pm 187$ & $0.067 \pm 0.033$ \\
\hline $\mathrm{V} 1121+2327$ & 562 & $5.24 \pm 0.37$ & $, 2.50)$ & 64 & 447 & $3 \pm 167$ & 0.043 & $1217 \pm 225$ & $50 \pm 0.031$ \\
\hline $0-1202$ & 562 & $6.35 \pm 1.15$ & $.47)$ & 297 & 318 & $5 \pm 132$ & & $795 \pm 163$ & $0.082 \pm 0$ \\
\hline $48.7+4456$ & .570 & $3.42 \pm 0.35$ & & 380 & 196 & & & $679 \pm 127$ & $0.068 \pm 0.039$ \\
\hline MACS-J2129.4 - 0741 & .570 & $9.17 \pm 0.90$ & $0,14.4,0.32,0.48,2.50)$ & 1199 & 639 & \pm 81 & & $1526 \pm 92$ & $0.066 \pm 0.009$ \\
\hline MS2053.7 - 0449 & 583 & $5.77 \pm 0.51$ & & & 357 & \pm 132 & 36 & $975 \pm 183$ & $0.065 \pm 0.033$ \\
\hline$-J 0647.7+7015$ & .584 & $12.79 \pm 1.59$ & 1.92) & 832 & 584 & $40 \pm 149$ & 27 & $1586 \pm 192$ & $0.061 \pm 0$. \\
\hline RXJ0956.0 + 4107 & .587 & $7.59 \pm 2.00$ & & 450 & 424 & $1046 \pm 332$ & $0.088 \pm 0.130$ & $1152 \pm 457$ & $0.072 \pm 0.122$ \\
\hline CL0542.8 - 4100 & & & & 714 & 539 & & & $984 \pm 164$ & \\
\hline RXJ1113.1 - 2615 & & $5.92 \pm 0.76$ & & 290 & 28 & $934 \pm 177$ & 58 & $1046 \pm 238$ & $30 \pm 0.049$ \\
\hline $\mathrm{J} 2302.8+0844$ & 734 & $8.39 \pm 1.35$ & $0.78)$ & 930 & 393 & $856 \pm 84$ & $0.090 \pm$ & $876 \pm 95$ & 0.026 \\
\hline $1137.5+6624$ & 0.782 & $6.87 \pm 0.52$ & $47,2.18)$ & 590 & 367 & $3 \pm 123$ & $89 \pm 0.040$ & $968 \pm 172$ & $0.075 \pm 0.037$ \\
\hline & 0.805 & $4.51 \pm 1.17$ & & 202 & 18 & & $0.042 \pm 0.026$ & $751 \pm 130$ & $0.037 \pm 0.021$ \\
\hline RXJ1350.0 + 6007 & 810 & $4.44 \pm 0.67$ & & 526 & 483 & $=146$ & $0.151 \pm 0.1$ & $660 \pm 219$ & $0.140 \pm 0.121$ \\
\hline RXJ1716.4 + 6708 & 0.813 & $7.04 \pm 0.81$ & $(1.000,0.254,1.5,0.67,0.30,1.79)$ & 565 & 408 & $844 \pm 124$ & $0.110 \pm 0.055$ & $904 \pm 166$ & $0.097 \pm 0.051$ \\
\hline RXJ0152.7 - 1357S & 0.828 & $9.43 \pm 2.35$ & $(0.367,0.533,3.6,0.35$ & 420 & 400 & $1162 \pm 248$ & $0.030 \pm 0.037$ & $1326 \pm 309$ & $0.022 \pm 0.026$ \\
\hline & & & & 848 & 599 & & & $1067 \pm 128$ & \\
\hline RXJ0152.7 - 1357N & 0.835 & $6.74 \pm 1.03$ & $(0.073,0.642,7.8,0.00,0.30,2.50)$ & 582 & 442 & $866 \pm 179$ & $0.117 \pm$ & $975 \pm 265$ & $0.095 \pm 0.094$ \\
\hline 1WGA-J1226.9 + 3332 & 0.890 & $12.14 \pm 1.38$ & $(0.077,0.257,29.1,0.00,0.46,0.87)$ & 677 & 497 & $1126 \pm 166$ & $0.085 \pm 0.040$ & $1222 \pm 226$ & $0.071 \pm 0.035$ \\
\hline CL1415.1 + 3612 & 1.030 & $6.93 \pm 0.74$ & $.30,2.50)$ & 425 & 318 & $857 \pm 93$ & $0.059 \pm 0.026$ & $960 \pm 121$ & $0.045 \pm 0.020$ \\
\hline RDCS-J0910 + 5422 & 1.106 & $6.43 \pm 1.41$ & $(0.147,1.000,3.4,0.58,0.36,1.20)$ & 230 & 201 & $576 \pm 144$ & $0.117 \pm 0.092$ & $570 \pm 208$ & $0.116 \pm 0.096$ \\
\hline RDCS-J1252 - 2927 & 1.235 & $7.57 \pm 1.18$ & $(1.000,0.089,4.0,0.32,0.30,1.20)$ & 329 & 287 & $636 \pm 123$ & $0.079 \pm 0.061$ & $662 \pm 172$ & $0.073 \pm 0.061$ \\
\hline RDCS-J0849 + 4452 & 1.261 & $5.21 \pm 1.31$ & $(0.046,1.000,10.3,0.00,0.36,1.20)$ & 222 & 197 & $504 \pm 152$ & $0.103 \pm 0.100$ & $512 \pm 216$ & $0.099 \pm 0.103$ \\
\hline RDCS-J0848 + 4453 & 1.273 & $3.75 \pm 1.86$ & $(0.069,1.000,6.8,0.00,0.60,1.20)$ & 112 & 165 & $432 \pm 151$ & $0.052 \pm 0.295$ & $456 \pm 192$ & $0.047 \pm 0.436$ \\
\hline
\end{tabular}

Column (1): name; Col. (2): adopted redshift; Col. (3): best-fit gas temperature within $R_{\text {spec }}$; Col. (4): best-fit parameters of the gas density profile in Eq. (5) fitted over the radial range $0-R_{\text {spat }}$; Col. (5): outer radius $R_{\text {spat }}$ where the signal-to-noise ratio is above 2; Col. (6): radius $R_{\text {spec }}$ of the circular region adopted in the spectral fit; Cols. (7-8): $R_{500}$ and $f_{\text {gas }}$ estimated by assuming the temperature profile in Eq. (6); Cols. (9-10): $R_{500}$ and $f_{\text {gas }}$ estimated by assuming a constant temperature equal to $k T_{\text {gas }}$. A cosmology of $\left(H_{0}, \Omega_{\mathrm{m}}, \Omega_{\Lambda}\right)=\left(70 \mathrm{~km} \mathrm{~s}^{-1} \mathrm{Mpc}^{-1}, 0.3,0.7\right)$ is adopted here.

moving inwards. We consider the profiles from the deprojection of the background-subtracted surface brightness $S$, with error $\epsilon_{S}$, up to the radial limit, $R_{\text {spat }}$, beyond which $\left(S / \epsilon_{S}\right)<2$. The gas density profiles, normalized to the critical density at the cluster's redshift, are shown in Fig. 2. We note that the average profile measured in local $(z<0.3)$ clusters is definitely more peaked than the ones observed at higher redshift, with a central gas density that is a factor of 2 higher than in systems at $0.3<z<0.6$ and almost a factor of 5 higher than in objects at $z>0.6$. This follows recent evidence that cooling cores, at relativly higher central density, preferentially form later in time (e.g. Santos et al. 2008; Ettori \& Brighenti 2008).
To estimate the gas and total mass profiles, we fit the electron density profiles with a functional form adapted from Vikhlinin et al. (2006):

$$
\begin{aligned}
n_{\mathrm{e}}(r) & =a_{2} x_{0}^{-a_{3}} \times\left(1+x_{0}^{2}\right)^{-1.5 a_{4}+a_{3} / 2} \times\left(1+x_{1}^{a_{5}}\right)^{-a_{6} / a_{5}} \\
x_{0} & =r / a_{0} \\
x_{1} & =r / a_{1} .
\end{aligned}
$$

The number of free parameters is reduced according to the number of datapoints $N_{\text {dat }}$ available, in the following order of priority: $a_{5}=3$ (always), $a_{6}=1.2$ (when $6 \leq N_{\mathrm{dat}}<8$, otherwise free to vary between 0 and 2.5), $a_{4}=0.6\left(\right.$ when $\left.N_{\text {dat }}=5\right), a_{3}=0$ 
Table 2. Properties of the local cluster sample from Vikhlinin et al. (2006).

\begin{tabular}{ccccc}
\hline \hline Cluster & $z$ & $\begin{array}{c}k T_{\text {gas }} \\
\mathrm{keV}\end{array}$ & $\begin{array}{c}R_{500} \\
\mathrm{kpc}\end{array}$ & $\begin{array}{c}f_{\text {gas }} \\
\left(<R_{500}\right)\end{array}$ \\
\hline $\mathrm{A} 133$ & 0.057 & $4.15 \pm 0.07$ & $1027 \pm 38$ & $0.087 \pm 0.006$ \\
$\mathrm{~A} 1795$ & 0.062 & $6.10 \pm 0.06$ & $1320 \pm 47$ & $0.108 \pm 0.006$ \\
$\mathrm{~A} 2029$ & 0.078 & $8.46 \pm 0.09$ & $1419 \pm 32$ & $0.128 \pm 0.007$ \\
$\mathrm{~A} 478$ & 0.088 & $7.95 \pm 0.14$ & $1398 \pm 62$ & $0.125 \pm 0.011$ \\
$\mathrm{~A} 1413$ & 0.143 & $7.38 \pm 0.12$ & $1377 \pm 49$ & $0.112 \pm 0.007$ \\
$\mathrm{~A} 907$ & 0.160 & $5.96 \pm 0.08$ & $1149 \pm 32$ & $0.129 \pm 0.006$ \\
$\mathrm{~A} 383$ & 0.188 & $4.80 \pm 0.12$ & $983 \pm 34$ & $0.129 \pm 0.007$ \\
$\mathrm{~A} 2390$ & 0.230 & $8.90 \pm 0.17$ & $1489 \pm 50$ & $0.147 \pm 0.009$ \\
\hline
\end{tabular}

Column (1): name; Col. (2): adopted redshift; Col. (3): best-fit spectral temperature; Col. (4): $R_{500}$; Col. (5): gas mass fraction within $R_{500}$. A cosmology of $\left(H_{0}, \Omega_{\mathrm{m}}, \Omega_{\Lambda}\right)=\left(70 \mathrm{~km} \mathrm{~s}^{-1} \mathrm{Mpc}^{-1}, 0.3,0.7\right)$ is adopted here.

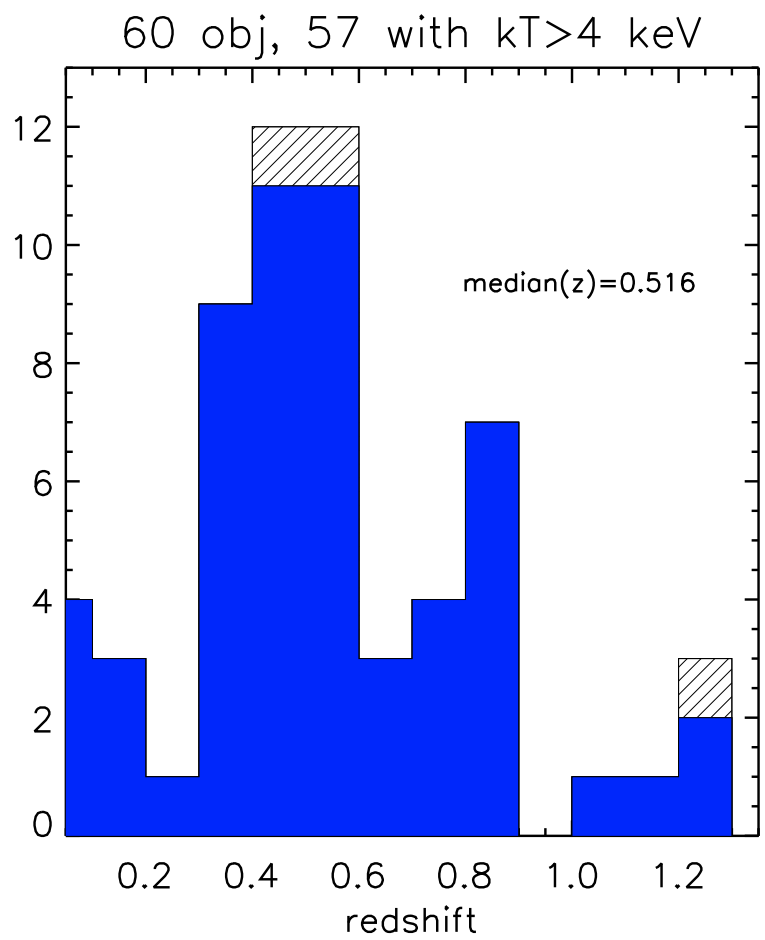

Fig. 1. Redshift distribution of the clusters in the whole sample (dashed region) and of the selected hottest objects (shaded regions).

(when $\left.N_{\text {dat }} \leq 4\right)$. The best-fit parameters that reproduce the electron density profiles up to the outer radial limit $R_{\text {spat }}$ are quoted in Table 1 . All the electron density profiles and the best-fit lines are shown in Fig. A.1.

The total and gas masses are then evaluated at $R_{\Delta}=R_{500}$ that describes the sphere within which the cluster overdensity with respect to the critical density is $\Delta=500$. This value of overdensity is maintained fixed in all the cosmologies studied and at any redshift. Under the assumption of isothermality, we calculate therefore

$$
\begin{aligned}
M_{\mathrm{tot}}(<r) & =-\frac{k T_{\mathrm{gas}} r}{\mu m_{\mathrm{u}} G} \frac{\mathrm{d} \log n_{\mathrm{e}}}{\mathrm{d} \log r}, \\
R_{500} & =\left(\frac{3 M_{\mathrm{tot}}\left(<R_{500}\right)}{4 \pi 500 \rho_{\mathrm{c}, \mathrm{z}}}\right)^{1 / 3}, \\
M_{\mathrm{gas}}(<r) & =\int_{0}^{R_{500}} 1.155 m_{\mathrm{u}} n_{\mathrm{e}}(r) 4 \pi r^{2} \mathrm{~d} r,
\end{aligned}
$$

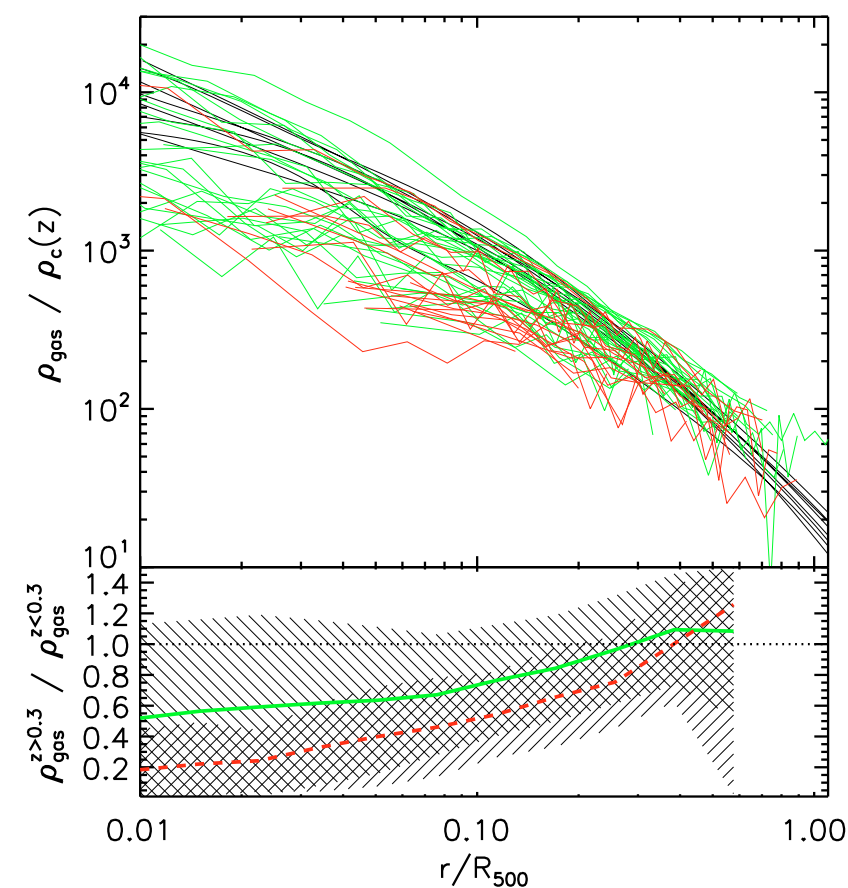

Fig. 2. Radial gas density profiles in units of the critical density at the cluster redshift. Green, black and red lines indicate objects at redshift $<0.3,0.3-0.6$ and $>0.6$, respectively. The bottom panel shows the ratio (and relative dispersion) of the mean density profiles observed in the redshift range $0.3-0.6$ (green line) and $z>0.6$ (red line) with respect to the local profile.

where 1.155 is the value associated to a cosmic mix of hydrogen and helium with 0.3 times solar abundance in the remaining elements with a relative contribution that follows Grevesse \& Sauval (1998), $\mu=0.600$ is the corresponding mean molecular weight, $m_{\mathrm{u}}=1.66 \times 10^{-24} \mathrm{~g}$ is the atomic mass unit and $\rho_{\mathrm{c}, \mathrm{z}}$ is the critical density at redshift $z$ and is equal to $3 H_{z}^{2} /(8 \pi G)$ with $H_{z}=H_{0} E(z)$ (see Eq. (2)). The gas mass fraction is then $f_{\text {gas }}\left(R_{\Delta}\right)=M_{\text {gas }}\left(<R_{\Delta}\right) / M_{\text {tot }}\left(<R_{\Delta}\right)$. The distribution of $R_{\text {spat }} / R_{500}$ and of $R_{\text {spec }} / R_{500}$ as a function of redshift is shown in Fig. 3.

All the errors are determined at $1 \sigma$ confidence level from the 16th and 84th percentile of the distribution of the values obtained by repeating the calculations 1000 times after a new surface brightness profile and gas temperature are considered by selecting normally-distributed random values according to the original measurements and their relative errors. A new total mass profile is then estimated and new $R_{500}$ and gas mass measurements are obtained by following Eqs. (5).

Our estimates of the gas fraction rely upon the determination of the total gravitating mass obtained under the assumption of an isothermal gas. To check the effect of the presence of a temperature profile on the measured total mass and, hence, gas fraction, we assume the expression for the temperature profile that Vikhlinin et al. (2006) showed to reproduce well the temperature gradients in nearby relaxed systems:

$T(r)=1.23 \frac{(x / 0.045)^{1.9}+0.45}{(x / 0.045)^{1.9}+1} \frac{T_{\text {spec }}}{\left[(x / 0.6)^{2}+1\right]^{0.45}}$,

where $x=r / R_{500}$ and the factor 1.23 comes from Eqs. (8) and (9) in Vikhlinin et al. (2006). Starting from the value of $R_{500}$ measured under the assumption of isothermality, we calculate (i) the temperature gradient; (ii) the total mass using the hydrostatic equilibrium equation $\left(M_{\mathrm{tot}} \propto T(r) \times r \times \mathrm{d} \log \left(n_{\mathrm{e}} T\right) / \mathrm{d} \log r\right)$; and 


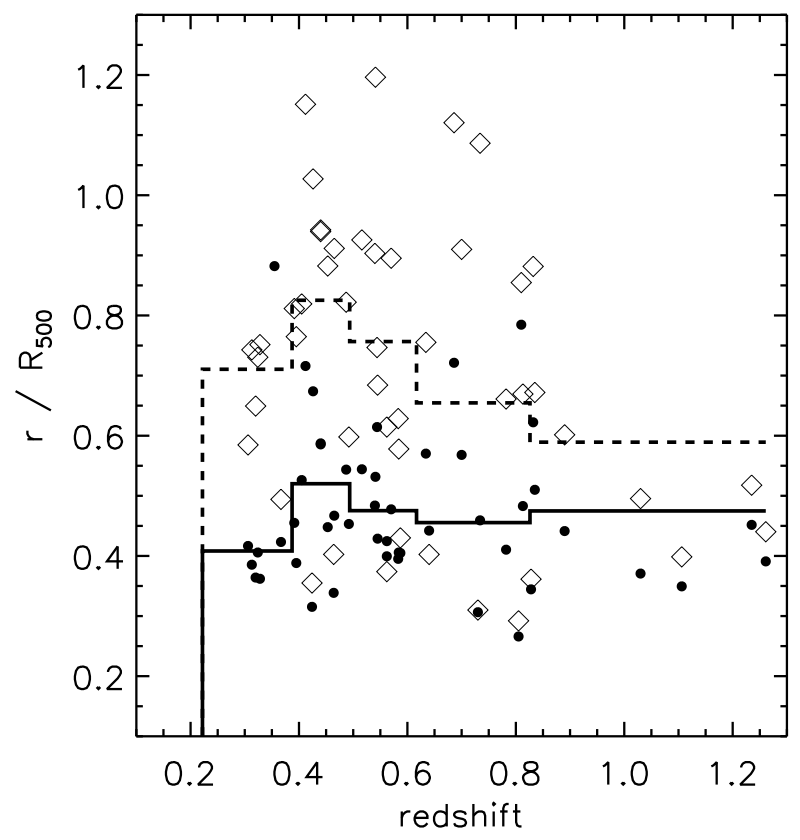

Fig. 3. Ratios between the maximum radial extension in the gas density profile, $R_{\text {spat }}$, and $R_{500}$ (diamonds) and the extraction radius of the circular region used to map the object in the spectral analysis, $R_{\text {spec }}$, and $R_{500}$ (dots). The dashed and solid lines show the average values of these ratios, $R_{\text {spat }} / R_{500}$ and $R_{\text {spec }} / R_{500}$ respectively, in bins of $\sim 10$ objects.

(iii) a new estimate of $R_{500}$. The calculations are repeated until $R_{500}$, that is evaluated from the total mass profiles and adopted in Eq. (6), converges. This happens after 2-6 iterations. At the newly estimated $R_{500}$, we measure the gas and total masses. We find that introducing a temperature profile decreases $R_{500}$ by 7 per cent, on average, and increases $f_{\text {gas }}\left(<R_{500}\right)$ by about 16 per cent with respect to the estimates obtained under the assumption of isothermality. The changes induced in the determination of $R_{500}$ and of $f_{\text {gas }}$ are shown in Fig. 4.

As a reference, for $\left(h_{70}, \Omega_{\mathrm{m}}=1-\Omega_{\Lambda}\right)=(1,0.3)$, in the overall sample of 52 objects at $z>0.3$, we measure $f_{\text {gas }, 500}=$ $(0.106,0.113,0.044)$ (median / mean / dispersion), when a gas temperature profile is assumed, and $(0.096,0.099,0.043)$, when a constant $k T$ is adopted.

In the following analysis, we make use of the estimates obtained with a temperature profile to limit the cosmological parameters for the case of reference, and leave to the discussion on the systematics the case with a constant temperature.

\subsection{Comparison with previous work}

We compare our results with recent determinations of the gas mass fraction obtained from a similar X-ray dataset.

Allen et al. (2008) presented an extensive cosmological study which uses the gas mass fraction in 42 hot $(k T>4 \mathrm{keV})$ relaxed clusters. When we consider the 16 objects in common with the present work, the mean (dispersion) of the ratios of the estimated $f_{\text {gas }}$ at $R_{2500}$ between their measurements and our estimates obtained by assuming a temperature profile is $1.14(0.24)$. Also within the scatter observed in this small sample used for comparison is the ratio between the spectroscopic measurements of the gas temperature $\left(T_{\text {Allen }} / T_{\text {this work }}=1.06, \mathrm{rms}=0.17\right)$.

We recall here that our gas temperatures are measured in spectra obtained to maximize the signal-to-noise ratio, including the contribution from the core emission. Any cool component in the core is expected to bias downwards the emission-weighted cluster temperature. We have checked the statistical effect of this bias by considering the sample of 90 clusters with temperatures $>4 \mathrm{keV}$ for which Maughan et al. (2007) obtained from Chandra observations a measure of the temperature both in the range $(0-1) R_{500}$ and in the range $(0.15-1) R_{500}$. We obtain a mean $T_{0.15-1} / T_{0-1}$ of 1.01 , with a dispersion of 0.14 , suggesting that, even though on average a cool core does not significantly affect the cluster temperature, the scatter in the distribution of the measured temperatures can be high.

Overall, these results suggest that some systematics (e.g., the background subtraction, the energy range adopted for spectral fitting; see discussion in Appendix of Balestra et al. 2007), of the order of 10-20 per cent and comparable to the mean statistical error of 10 per cent, affect the estimates of the global temperatures. These uncertainties propagate also to the measurements of the gas mass fraction. To assess the role of these errors on the robustness of our constraints, we investigate in Sect. 5.4 how our results depend upon the sample selected according to the measured relative error on the gas mass fraction, an error that is propagated from the uncertainties on $T_{\text {gas }}$.

\section{The cluster gas fraction as cosmological probe: the method}

We start with a grid of values for the cosmological parameters we want to investigate, specifically $\Omega_{\mathrm{m}}$ and $\Omega_{\Lambda}$ or $w$. We compute then

$\chi^{2}=\sum_{i=1}^{N_{\mathrm{dat}}} \frac{\left(f_{\mathrm{bar}, i} / b_{i}-\Omega_{\mathrm{b}} / \Omega_{\mathrm{m}}\right)^{2}}{\epsilon_{\mathrm{bar}, i}^{2} / b_{i}^{2}+\left(f_{\mathrm{bar}, i} \epsilon_{b_{i}} / b_{i}^{2}\right)^{2}+\epsilon_{\Omega_{\mathrm{b}}}^{2} / \Omega_{\mathrm{m}}^{2}}$.

In the above equation, we use the following definitions:

1. $f_{\text {bar }}=f_{\text {gas }}+f_{\text {cold }}$, where the gas mass fraction $f_{\text {gas }}$ is directly measured from our X-ray observation and depends upon the cosmological parameters through the angular diameter distance, $d_{\text {ang }}$, defined in Eq. (1), being $f_{\text {gas }}=M_{\text {gas }} / M_{\text {tot }} \propto$ $n_{\text {gas }} R^{3} / R \propto d_{\text {ang }}^{5 / 2} / d_{\text {ang }} \propto d_{\text {ang }}\left(\Omega_{\mathrm{m}}, \Omega_{\Lambda}, w\right)^{3 / 2}$ (see Fig. 5 for the relative dependence upon different cosmologies), while the mass fraction in cold baryons, $f_{\text {cold }}=M_{\text {cold }} / M_{\text {tot }}$, is estimated statistically as described in the next subsection;

2 . the error on $f_{\mathrm{bar}}, \epsilon_{\mathrm{bar}}$, is the sum in quadrature of the uncertainties on $f_{\text {gas }}, f_{\text {star }}$ and on the assumed value of Hubble's constant $H_{0}$ (see below) propagated through the following dependence: $f_{\text {gas }} \propto H_{0}^{-1.5}$ and $f_{\text {cold }} \propto H_{0}^{-1}$;

3 . the depletion parameter $b$ (with error $\epsilon_{b}$ ) represents the fraction of cosmic baryons that fall in the cluster dark matter halo and is estimated from hydrodynamical simulations as discussed in Sect. 4.2;

4. we assume $\Omega_{\mathrm{b}} h_{70}^{2}=0.0462 \pm 0.0012$ (error at $1 \sigma$ level) from the best-fit results of the joint analysis in Komatsu et al. (2008) of (i) the power spectrum of the temperature anisotropy measured from the Wilkinson Microwave Anisotropy Probe five year data release (WMAP 5-year, Dunkley et al. 2008); (ii) a combined set of magnitudes of type Ia supernovae (Riess et al. 2007; Astier et al. 2006; Wood-Vasey et al. 2007); (iii) the Baryon Acoustic Oscillations measured in a survey of galaxies at $z=0.2$ and $z=0.35$ (Percival et al. 2007).

We note that the recent compilation of the best-fit results from the Primordial Nucleosythesis calculations on $\Omega_{\mathrm{b}}$ provides comparable constraints (see, e.g., Steigman 2006, 

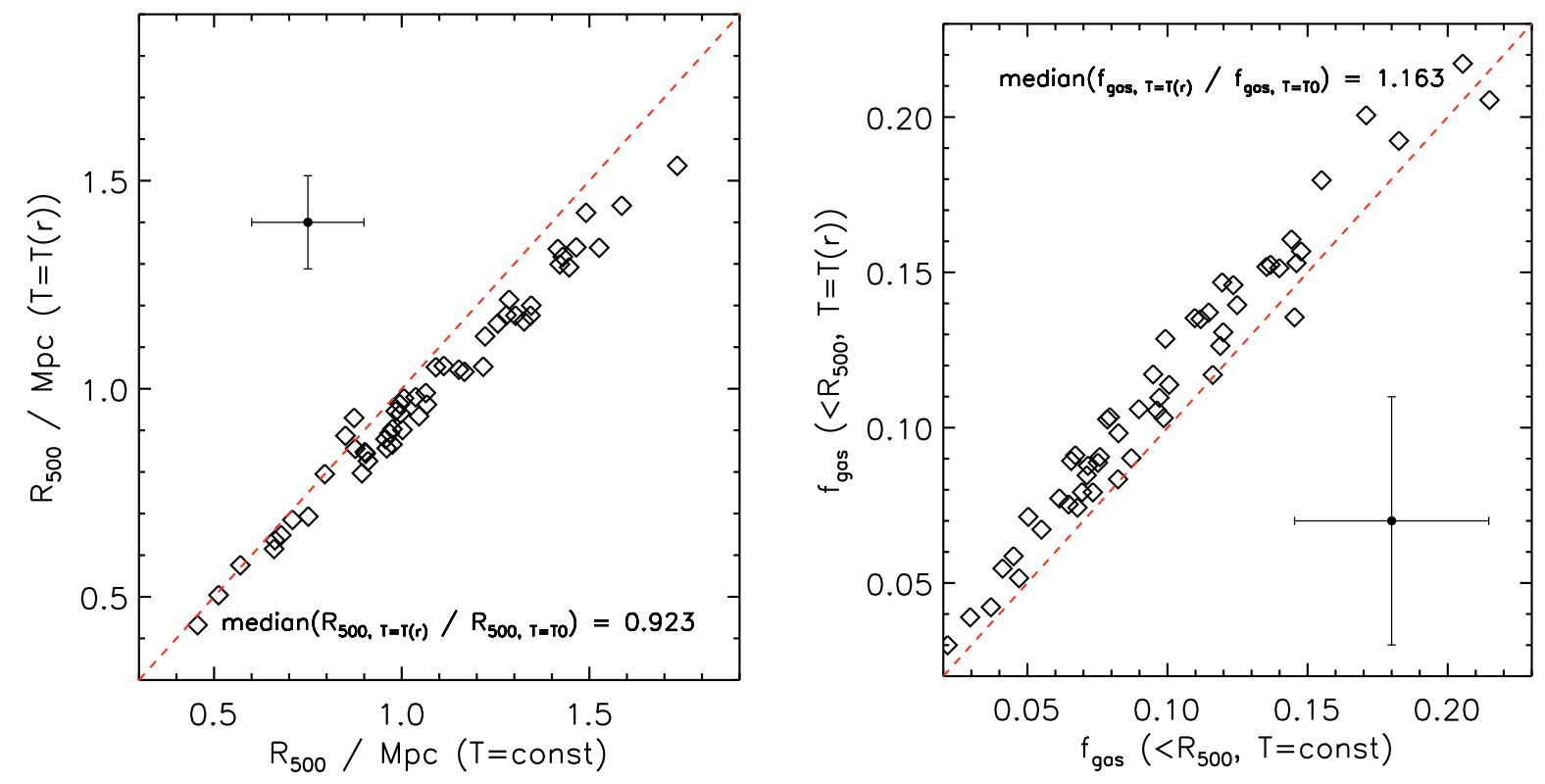

Fig. 4. Changes in estimates of $R_{500}$ and $f_{\text {gas }}$ once the temperature profile in Eq. (6) is assumed. Median relative error bars are shown.

page 34: $\left.\Omega_{\mathrm{b}} h_{70}^{2}=0.0454 \pm 0.0045\right)$. We consider the WMAP 5-year limits in the following analysis.

We adopt a present-day Hubble's constant of $H_{0}=72 \pm$ $8 \mathrm{~km} \mathrm{~s}^{-1}$ (error at $1 \sigma$ level) measured in the Hubble Space Telescope Key Project by Freedman et al. (2001). This value, assumed also as a Gaussian prior in the joint cosmological analysis from Komatsu et al. (2008), is in agreement with their final result of $H_{0}=70.1 \pm 1.3 \mathrm{~km} \mathrm{~s}^{-1}$, but is marginally in conflict with the value of $H_{0}=62.3 \pm$ 1.3 (random) \pm 5.0 (systematic) $\mathrm{km} \mathrm{s}^{-1} \mathrm{Mpc}^{-1}$ recently determined from type Ia supernovae calibrated with Cepheid variables in the nearby galaxies that hosted them (Sandage et al. 2006). We make use of these estimates of $H_{0}$ in Sect. 5 , where we discuss the robustness of our cosmological constraints.

For a set of cosmological parameters $\left\{\Omega_{\mathrm{m}}, \Omega_{\Lambda}, w\right\}$, the gas mass, the total mass and the critical density $\rho_{c, z}$ are evaluated by considering all their cosmological dependencies. A new gas fraction for each cluster in the selected sample is then estimated at the fixed overdensity of $\Delta=500$ following Eqs. (5).

\subsection{The stellar mass fraction}

The stellar mass fraction, $f_{\text {star }}=M_{\text {star }} / M_{\text {tot }}$, cannot be computed individually for each single object for which most of the information of, e.g., the luminosity function of many clusters at medium and high redshift is lacking. Therefore, a statistical approach is generally used by estimating $f_{\text {star }}$ as a function of other observed quantities for which it has been possible to confirm a robust correlation on a small and local sample of galaxy clusters.

From the measurements of the optical luminosity and Rosat X-ray surface brightness in Coma, White et al. (1993) found $f_{\text {star }}=0.18( \pm 0.05) f_{\text {gas }}$. Arnaud et al. (1992) quote $f_{\text {star }}=$ $0.17( \pm 0.03) f_{\text {gas }}$ when their values are converted using a mass-tolight ratio for stars in the $V$ band of $6(M / L) \odot$ (see also Voevodkin \& Vikhlinin 2004). Similar results have been obtained from Fukugita et al. (1998) by estimating the global budget of cosmic baryons. In the following analysis, we will refer to these very similar recipes as $f_{\text {star, } \mathrm{W}}=0.18( \pm 0.05) f_{\text {gas }}$.

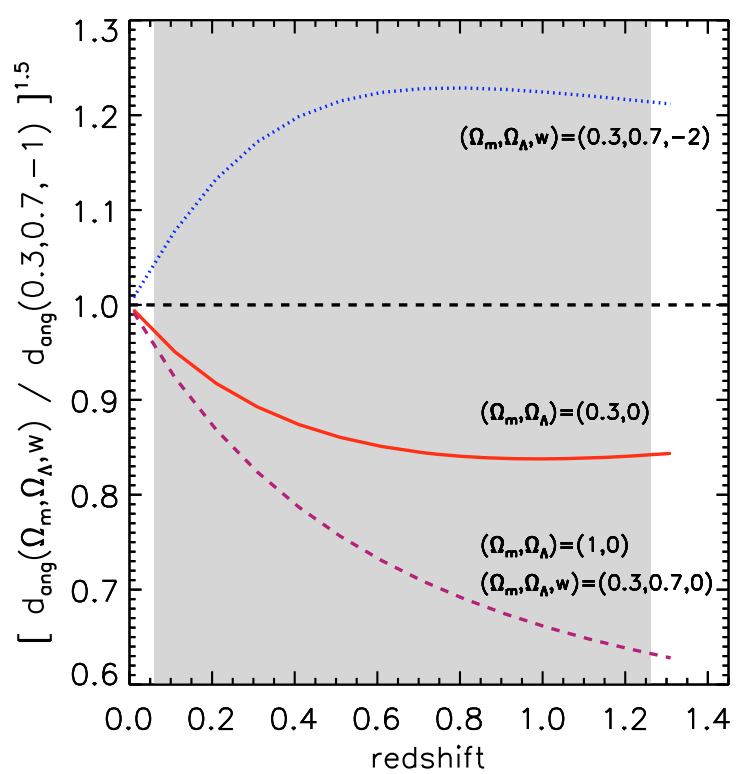

Fig. 5. Sensitivity of the cluster baryon fraction method to the variation of the cosmological parameters. The shaded region indicates the redshift range considered in the present study.

Lin et al. (2003) use the near-infrared light as a tracer of the total stellar mass within cluster galaxies. They find a good correlation between the stellar mass fraction obtained from the $K$-band luminosity function measured using the Two Micron All Sky Survey (2MASS) data and X-ray properties of 27 nearby systems. Considering that their results apply to all the sample that spans a gas temperature between 2 and $9 \mathrm{keV}$ and that they rely on total mass estimates obtained from scaling relations taken from the literature, we use their measurements of $M_{\text {star }}$ and their assumed $T_{\text {gas }}$ to recover an empirical relation valid for the clusters with $T_{\text {gas }}>4 \mathrm{keV}$ that we will consider in our analysis. We obtain that, for these 10 very massive clusters, the ratio $M_{\text {star,500 }} / T_{\text {gas }}^{1.5}$ is almost independent of the temperature itself and is equal to $5.09( \pm 1.73) \times 10^{12} M_{\odot}(5 \mathrm{keV})^{-1.5}$ (weighted mean 


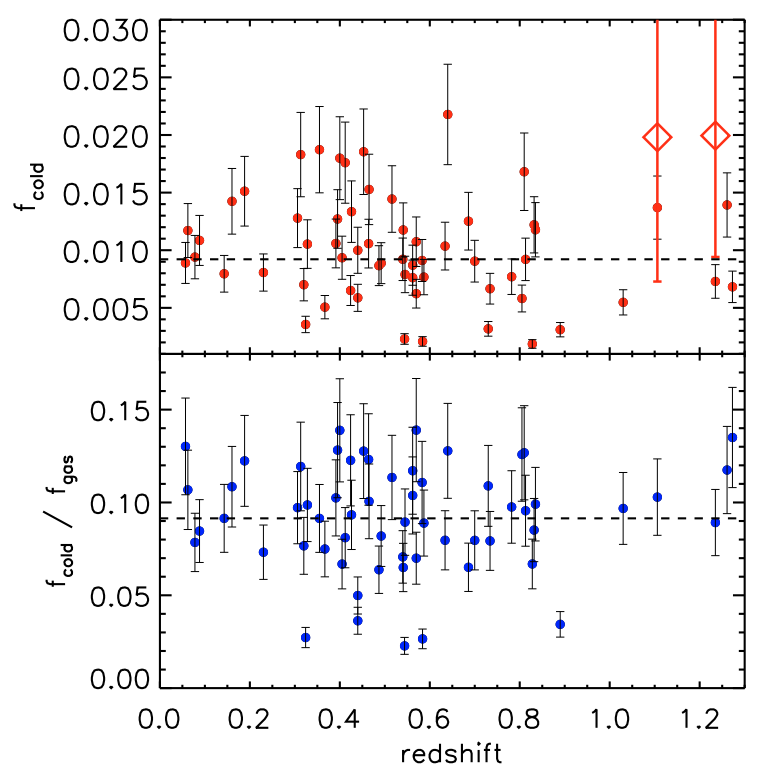

Fig. 6. Stellar mass fractions (cf. Eq. (8)) and ratios between their values and the estimated gas mass fraction at $R_{500}$ as function of redshift. Dashed lines indicate the median value: $f_{\text {cold }}=0.009$ and $f_{\text {cold }} / f_{\text {gas }}=0.091$. The two diamonds indicate the values for RDCSJ0910 and RDCS-J1252 with the stellar masses estimated from the nearinfrared luminosity function in Strazzullo et al. (2006). A cosmology of $\left(H_{0}, \Omega_{\mathrm{m}}, \Omega_{\Lambda}\right)=\left(70 \mathrm{~km} \mathrm{~s}^{-1} \mathrm{Mpc}^{-1}, 0.3,0.7\right)$ is adopted here.

and dispersion after propagation of the errors on both $M_{\mathrm{star}, 500}$, for which we assume the relative error on the estimates of the luminosity, and $T_{\text {gas }}$ ). The estimated stellar masses are then added to the measured gas masses to evaluate the total baryon mass. We will refer to $f_{\text {star, } \mathrm{L}}$ to indicate this method calculating the stellar contribution to the total baryon budget.

An additional component of the cold baryon budget is the intracluster light (ICL) at very low surface brightness, making the total cold baryonic content of galaxy clusters equal to $f_{\text {cold }}=f_{\text {star }}+f_{\text {ICL }}$. Numerical simulations (e.g. Willman et al. 2004; Murante et al. 2004, 2007) suggest that the ICL mostly originates from the merging processes taking place during the assembly of massive cluster galaxies. Consistently with observational results for the Virgo cluster (Feldmeier et al. 2004), simulations predict $f_{\mathrm{ICL}} \approx 0.2 f_{\text {star }}$ in lower mass systems, with an increasing fraction up to $f_{\mathrm{ICL}} \approx f_{\text {star }}$ in $10^{15} M_{\odot}$ clusters (e.g. Lin \& Mohr 2004).

Gonzalez, et al. (2007) have presented a census of the baryons in local systems with total masses in the range $6 \times$ $10^{13} M_{\odot}-10^{15} M_{\odot}$. Including the ICL luminosity well mapped within $300 \mathrm{kpc}$, but probably underestimated in the outskirts if there is any significant contribution at larger radii, they found a well defined correlation between the stellar mass fraction and the total mass at $\Delta=500$ in the form of $f_{\text {cold }}=(0.009 \pm$ $0.002)\left(M_{500} / 10^{15} M_{\odot}\right)^{-(0.64 \pm 0.13)}$.

Recently, Lagana et al. (2008) discussed in detail the baryonic content of five massive galaxy clusters, including an ICL contribution. They conclude that the stellar-to-gas mass ratio within $R_{500}$ anti-correlates slightly with the gas temperature and can be expressed through the relation

$f_{\text {cold }}=f_{\text {star }}+f_{\text {ICL }}=\left(0.18-0.012 T_{\text {gas }}\right) f_{\text {gas }}$,

where $T_{\text {gas }}$ is measured in $\mathrm{keV}$. For clusters with $T_{\text {gas }}>4 \mathrm{keV}$, that is one of our selection criteria, $f_{\text {cold }} / f_{\text {gas }}<0.12$. These values lie on the lower end of the stellar mass distribution presented in the above-mentioned work, whereas $f_{\text {star,W }}$ represents an upper limit. However, considering that the analysis has been performed self-consistently for the baryonic component for a sample of massive galaxy clusters that well fit the properties of the objects studied in our work, we adopt this correlation between $f_{\text {cold }}$ and $f_{\text {gas }}$ and propagate the estimated $20 \%$ uncertainty on the $f_{\text {cold }}$ value.

As a reference, for $\left(h_{70}, \Omega_{\mathrm{m}}=1-\Omega_{\Lambda}\right)=(1,0.3)$, we obtain for the selected sample of massive objects $f_{\text {cold }, 500}=$ $(0.091 / 0.090 / 0.027) f_{\text {gas,500 }}$ (median / mean / dispersion; see Fig. 6) which represents about $10 \%$ of the total cluster baryon budget (see discussion in Sect. 5.5).

We study further the effect of (i) a different recipe to evaluate $f_{\text {star }}$; and (ii) the possibility of a larger, up to 20 per cent, contribution of the ICL, when we discuss the robustness of our results to some systematic errors in Sect. 5.4.

\subsection{The depletion parameter}

The depletion parameter $b=f_{\mathrm{bar}} /\left(\Omega_{\mathrm{b}} / \Omega_{\mathrm{m}}\right)$ indicates the amount of cosmic baryons that are thermalized within the cluster potential. When measured over well-representative regions of a galaxy cluster, this value tends to be lower than unity because not all the accreting shock-heated baryons relax within the dark matter halo. However, the depletion parameter can presently only be inferred from numerical simulations, where both the input cosmic baryons are known and the amount of them accreting into the cluster dark matter halo can be traced. For instance, Eke et al. (1998) find in their smoothed particle hydrodynamics non-radiative simulations that the gas fraction within $R_{\text {vir }}$ is, on average, 87 per cent of the cosmic value. On the other side, recent work with grid-based numerical codes estimates that a larger amount of baryons are captured in the cluster potential (e.g. Kravtsov et al. 2005). In a set of SPH and Eulerian simulations of a single cluster presented in the Santa Barbara Comparison Project, Frenk et al. (1999) measure at the virial radius $b=0.92 \pm 0.07$. In Ettori et al. (2006), we analyze the $b$ parameter in a set of four massive $\left(M_{200}>10^{15} h^{-1} M_{\odot}\right.$ at $z=0$ ) galaxy clusters simulated by using the Tree+SPH code GADGET-2. We consider here the distribution of the values of $b\left(<R_{500}\right)$ measured in two sets of hydrodynamical simulations, one with gravitational heating only, the other including radiative processes, such as cooling and star formations with feedback provided from weak winds (Fig. 7). We measure $b\left(<R_{500}\right)=$ $0.874 \pm 0.023$ at $z=0$ and $b\left(<R_{500}\right)=0.947 \pm 0.037$ at $z=1$ in the first set, and $b\left(<R_{500}\right)=0.920 \pm 0.026$ at $z=0$ and $b\left(<R_{500}\right)=0.961 \pm 0.028$ at $z=1$, when cooling and feedback are considered. If we average over the redshift range $0-1$, we obtain $b\left(<R_{500}\right)=0.940 \pm 0.023$, or, by fitting a polynomial at $1 \mathrm{st}$ order in redshift, $b\left(<R_{500}\right)=0.923( \pm 0.006)+0.032( \pm 0.010) z$. Considering that the four objects under consideration reached $M_{200}>10^{15} \mathrm{~h}^{-1} M_{\odot}$ at $z=0$ after they accreted mass by a factor 2-6 from $z=1$, we adopt two values for the depletion parameter, encompassing the results of our simulated dataset (see Fig. 7):

$b_{500}=0.874 \pm 0.023$,

that is related to the most massive systems, subjected only to the gravitational heating, in the studied simulation and, thus, particularly appropriate for the high-temperature objects that are analyzed in the present work over radial regions, beyond their cores, where radiative processes (like cooling, star formation, 


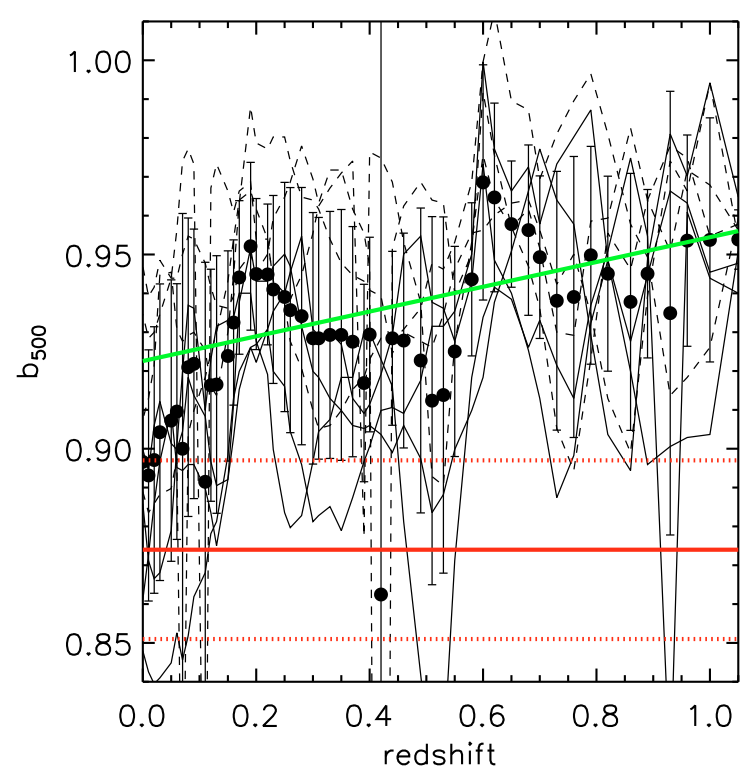

Fig. 7. Depletion parameter as a function of redshift as measured in cosmological hydrodynamical simulations in Ettori et al. (2006). The solid and dashed lines show results for non radiative simulations and for radiative simulations, which include star formation and galactic winds, respectively. These estimates are averaged over simulations of four massive clusters with $M_{200}>10^{15} M_{\odot} / h$ at $z=0$. The dots and errorbars indicate the average $b_{500}$ and the scatter around it. The red solid line indicates the best-fit constant value $b_{500}$ estimated at $z=0$ in non-radiative simulations (dotted lines show the dispersion around the mean). The green solid line represents $b_{500, z}$ of Eq. (10) adopted in our analysis.

stellar feedback) are not expected to influence significantly the ICM physiscs;

$b_{500, z}=0.923( \pm 0.006)+0.032( \pm 0.010) z$,

that is the quantity measured when all the simulated dataset are considered over the redshift range of interest.

\section{Results on the cosmological parameters}

We measure the gas mass fraction at $R_{500}$ in $52 z>0.3$ objects with a median relative error $\epsilon_{\text {gas }}$ of 36 per cent $(1 \sigma)$. In the local sample, the relative error is about 6 per cent.

In the following analysis, we focus on the most massive objects present in our sample to work under the ideal hypothesis that the physics of the X-ray emitting plasma is only determined by the gravitational collapse. To define a sample of massive systems whose baryon content is expected to be representative of the cosmic budget, we select the 49 objects at $z>0.3$ with $T_{\text {gas }}>4 \mathrm{keV}$ (see Fig. 1 and Table 1). We also consider the 8 galaxy clusters with $T_{\text {gas }}>4 \mathrm{keV}$ in the local sample of Vikhlinin et al. (2006; see Table 2)

\subsection{Constraints on $\Omega_{\mathrm{m}}$ and $\Omega_{\Lambda}$}

We assume hereafter that $\Omega_{\mathrm{b}} h_{70}^{2}=0.0462 \pm 0.0012, H_{0}=$ $72 \pm 8 \mathrm{~km} \mathrm{~s}^{-1} \mathrm{Mpc}^{-1}, b_{500}=0.874( \pm 0.023)$ and $f_{\text {cold }}=$ $\left(0.18-0.012 T_{\text {gas }}\right) f_{\text {gas }}$, with an associated 20 per cent relative error (at $1 \sigma$ level). From the estimated mean gas mass fraction within $R_{500}$ of 0.11 (see Sect. 3 ), the resulting matter density parameter is expected to measure (see Eq. (7))

$\Omega_{\mathrm{m}}=\frac{b \Omega_{\mathrm{b}}}{f_{\text {gas }}+f_{\text {cold }}} \approx 0.32$.
To make extensive use of the information present in the gas mass fraction distribution, we minimize Eq. (7) for a grid of values of $\left\{\Omega_{\mathrm{m}}, \Omega_{\Lambda}\right\}$ that satisfy the condition $\Omega_{\mathrm{m}}+\Omega_{\Lambda}+\Omega_{k}=1(w=-1)$. We obtain the best-fit results presented in Fig. 8 and quoted here at the $1 \sigma$ level of confidence on one single parameter $\left(\chi^{2}-\chi_{\min }^{2} \leq\right.$ 1) after marginalization over the others:

$\Omega_{\mathrm{m}}=0.35_{-0.04}^{+0.03}$

$\Omega_{\Lambda}=0.59_{-0.56}^{+0.46}$.

The total $\chi^{2}$ is 58.9 for $57-2$ degrees of freedom (see Fig. 10).

These results change slightly once the recent determination of Hubble's constant, $H_{0}=62.3 \pm 1.3$ (random) \pm 5.0(systematic) $\mathrm{km} \mathrm{s}^{-1} \mathrm{Mpc}^{-1}$ by Sandage et al. (2006), is adopted. With a $\chi^{2}=66.5$, we obtain

$\Omega_{\mathrm{m}}=0.37_{-0.04}^{+0.04}$
$\Omega_{\Lambda}=0.63_{-0.48}^{+0.46}$.

More stringent results are obtained when tighter constraints on Hubble's constant provided with a joint-fit with the cosmic microwave temperature anisotropies are considered. For example, by using the best-fit values from WMAP 5-year data analysis by Komatsu et al. $\left(2008 ; H_{0}=70.1 \pm 1.3 \mathrm{~km} \mathrm{~s}^{-1} \mathrm{Mpc}^{-1}\right.$, $\Omega_{\mathrm{b}}=0.0462 \pm 0.0015$; labelled "WMAP5" in Table 3), we obtain

$\Omega_{\mathrm{m}}=0.32_{-0.02}^{+0.03}$

$\Omega_{\Lambda}=1.01_{-0.28}^{+0.20}$,

but with a $\chi^{2}=148.9$ mainly due to the propagation of the relative error on the Hubble's constant value that is smaller than in our reference case ( 2 per cent vs. 11 per cent).

\subsection{Constraints on the equation of state of the dark energy}

We investigate the constraints that the cluster baryon fraction can place on the equation of state of the dark energy, $w=P / \rho$, where $P$ and $\rho$ are the pressure and density of the dark energy component, respectively. We consider both the case $w=$ constant and $w=w\left(w_{0}, w_{1}\right)$ as in Eq. (3). We present our reference results in Fig. 9.

Under the assumption of a flat Universe $\left(\Omega_{\mathrm{m}}+\Omega_{\Lambda}=1\right)$, the best-fit values for our reference model are $\left(\chi^{2}=58.9\right.$; $1 \sigma$ level of confidence on one single parameter):

$$
\begin{aligned}
\Omega_{\mathrm{m}} & =0.32_{-0.05}^{+0.04} \\
w & =-1.1_{-0.45}^{+0.60}
\end{aligned}
$$

No significant limits are obtained for an evolving $w$ (see Eq. (3)), with $w_{0}=-1.0_{-0.7}^{+0.7}$ and no constraints at $1 \sigma$ level on $w_{1}$ in the range $(-3,3)$.

Similarly to the case for $\Omega_{\Lambda}$, changes in the assumed prior distributions induce appreciable differences in the central values of the best-fit parameters. As shown in Fig. 11, these differences are however always within $2 \sigma$ for $w$, even in the most extreme cases, e.g. not considering the local $(z<0.3)$ objects or including in the analysis only the hottest $(k T>8 \mathrm{keV})$ clusters. For the measurements of $\Omega_{\mathrm{m}}$, the behaviour is the same as discussed in the previous section.

\subsection{Do the data require $\Omega_{\Lambda}>0$ ?}

Considering our best-fit results presented in Sect. 5.1, we obtain that $\Omega_{\Lambda}>0$ with a significance in the range $1-3.6 \sigma$ (see, e.g., 

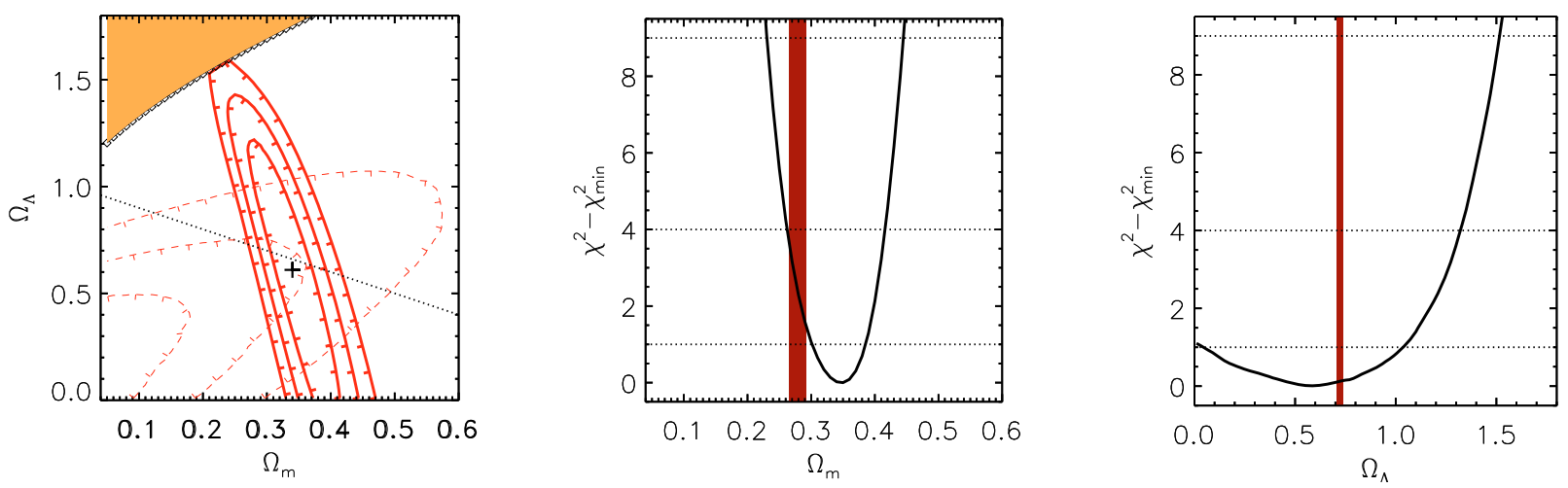

Fig. 8. Constraints in the $\Omega_{\mathrm{m}}-\Omega_{\Lambda}$ plane $(w=-1)$ provided from the sample of 57 objects. (Left) Likelihood contours at 1,2 and $3 \sigma\left(\Delta \chi^{2}=\right.$ $2.30,6.17,11.8$, respectively, for 2 degrees of freedom) for the constraints obtained through Eq. (7) (solid lines) and Eq. (16) by using the gas fraction evolution only (dashed lines). (Middle) Marginalized probability distribution for $\Omega_{\mathrm{m}}$. (Right) Marginalized probability distribution for $\Omega_{\Lambda}$. The shaded regions show the constraints from the joint cosmological analysis of CMB+SnIa+BAO data in Komatsu et al. (2008).
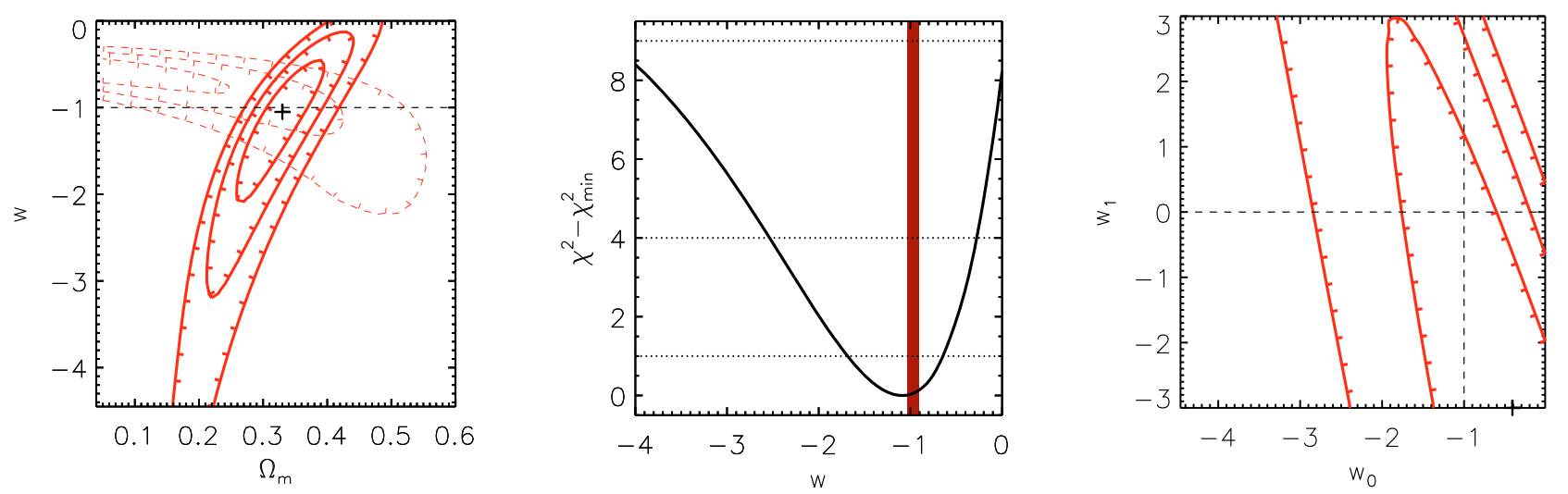

Fig. 9. (Left) Constraints in the $\Omega_{\mathrm{m}}-w$ plane $\left(\Omega_{\mathrm{m}}+\Omega_{\Lambda}=1\right)$ plane provided from the sample of 57 objects. Likelihood contours at 1,2 and $3 \sigma$ $\left(\Delta \chi^{2}=2.30,6.17,11.8\right.$, respectively, for 2 degrees of freedom) for the constraints obtained through Eq. (7) (solid lines) and Eq. (16) by using the gas fraction evolution only (dashed lines). (Middle) Marginalized probability distribution on $w$ overplotted on the best-fit constraints obtained from the joint cosmological analysis of $\mathrm{CMB}+\mathrm{SnIa}+\mathrm{BAO}$ data in Komatsu et al. (2008). (Right) Constraints in the $w_{0}-w_{1}$ plane from the definition adopted in Eq. (3).

Table 3). In particular, for our reference case (Fig. 8), our dataset requires a $\Delta \chi^{2}$ of about 1 when $\Omega_{\Lambda}$ is fixed to zero (see Fig. 10). Therefore, the baryonic mass fraction in galaxy clusters alone can only provide marginal evidence for a no null contribution from a dark energy component. This contribution is mainly due to the assumption that the gas mass fraction does not evolve with redshift in the "correct" cosmology. We can write it as

$\chi^{2}=\sum_{i=1}^{N_{\mathrm{dat}}} \frac{\left(f_{\mathrm{gas}, i}-\overline{f_{\mathrm{gas}}}\right)^{2}}{\epsilon_{\mathrm{gas}, i}^{2}+\epsilon_{\bar{f}}^{2}}$

where $f_{\mathrm{gas}, i}$ and $\epsilon_{\mathrm{gas}, i}$ are the single gas mass fraction measurements with the relative errors, $\overline{f_{\text {gas }}}$ and $\epsilon_{\bar{f}}$ are the mean and the error on the mean of the $f_{\text {gas }, i}$ values. In Fig. 8 , the likelihood contours are overplotted on the best-fit results. From these plots, it is possible to appreciate the different role played by the two assumptions made to define the "correct" cosmology: while the condition that the gas fraction is constant with look-back time marginally influences the value of $\Omega_{\mathrm{m}}$ but, at the same time, significantly tilts the contours favouring an $\Omega_{\Lambda}>0$ for $\Omega_{\mathrm{m}} \sim 0.35$, the condition that the cluster baryonic fraction is representative of the cosmic value has the greatest statistical weight, collapsing the probability around the best-fit value of $\Omega_{\mathrm{m}} \approx 0.35$.

\subsection{Robustness to systematic errors}

In this section, we investigate how our cosmological constraints are affected by potential systematic errors. To evaluate this, we repeat the analysis described in the previous sections and apply it to several different subsets defined by changing one of the criteria adopted for the selection of the reference sample of 57 hot galaxy clusters.

First of all, we do not observe any dependence of $f_{\text {gas }}$ on both temperature and metallicity in our reference sample: once objects with $T_{\text {gas }}>4 \mathrm{keV}$ are selected, the Spearman's rank correlation is about 0.1 implying an underlying correlation significant at less than the $1 \sigma$ confidence level.

Moreover, we notice that the estimated gas mass fraction within $R_{2500}$ is about 75 per cent of the value measured at $R_{500}$ (mean and standard deviation after 1000 bootstrap resampling $=75 \pm 2$; median $=73$ per cent), confirming its increased value outwards.

As shown in the plots of Fig. 11 (and summarized in Table 3), the largest uncertainties are related to the assumed prior on the temperature profile. By using the values obtained by assuming an isothermal ICM (see $\hat{f}_{\text {gas }}$ in Table 1 ), and applying the method described in Sect. 4, we obtain (total $\chi^{2}=75.5 ; \Omega_{\mathrm{m}}+\Omega_{\Lambda}+\Omega_{k}=$ $1, w=-1)$

$\Omega_{\mathrm{m}}=0.40_{-0.02}^{+0.03}$ 


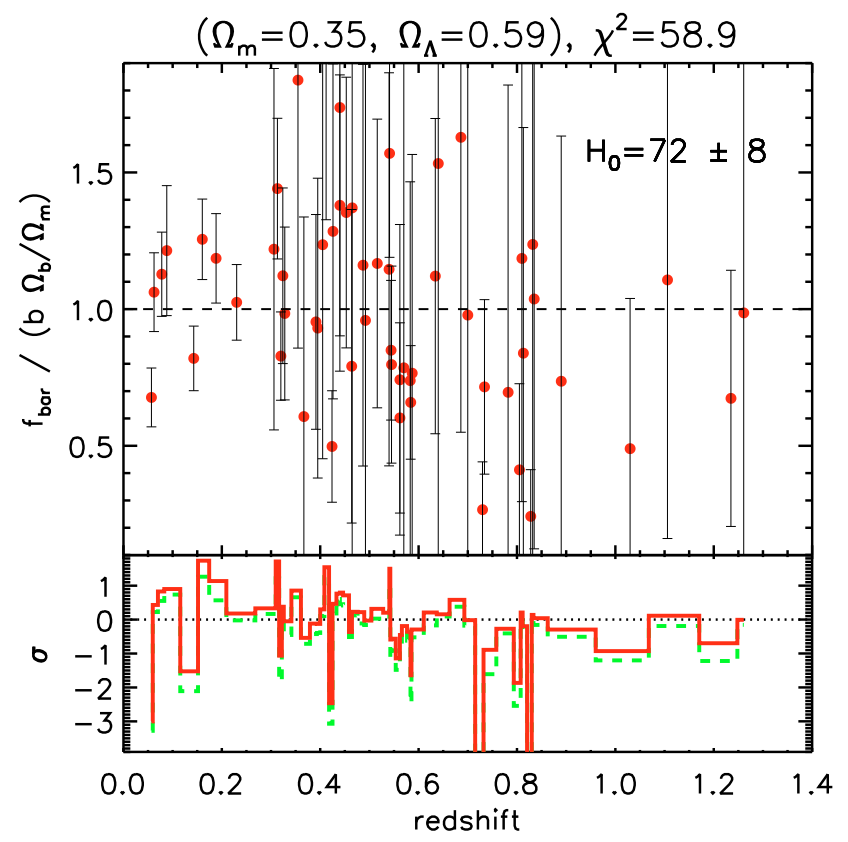

Fig. 10. Distribution of the measured baryon fractions in the sample of 57 objects ( 49 of which are at $z>0.3$ ) with $T_{\text {gas }}>4 \mathrm{keV}$. The error bars at $1 \sigma$ include all the components listed in Sect. 4 . The best-fit results of the cosmological model used as the reference are assumed. In the lower panel, the residuals with respect to the assumed value of $f_{\text {bar }} / b \times \Omega_{\mathrm{m}} / \Omega_{\mathrm{b}}=1$ are shown (solid line). The dashed line refers to the distribution obtained by fixing all the cosmological parameters to the best-fit results and assuming $\Omega_{\Lambda}=0$.

$$
\begin{aligned}
& \Omega_{\Lambda}<0.46, \\
& \text { and }\left(\chi^{2}=76.3 ; \Omega_{\mathrm{m}}+\Omega_{\Lambda}=1\right) \\
& \Omega_{\mathrm{m}}=0.29_{-0.05}^{+0.08} \\
& \quad \omega<-0.8 .
\end{aligned}
$$

The total $\chi^{2}$ shows a significant increase $\left(\Delta \chi^{2}=17\right)$, suggesting a poorer representation of the distribution of $f_{\text {gas }}$ and pointing out the necessity for a robust and reliable determination of the temperature profiles in galaxy clusters, possibly out to the region where the gas mass fraction measurements are more representative of the cluster baryon content $\left(r \gtrsim R_{2500} \approx 0.3 R_{200}\right)$.

The assumption on the stellar mass fraction does not significantly affect the estimates of the cosmological parameters. As discussed in Sect. 4.1, the stellar mass fraction that we adopt here from Lagana et al. (2008) lies on the lower end of the distribution available in the literature. By assuming instead $f_{\text {cold }} / f_{\text {gas }}=0.18$, independently from the cluster mass, as generally done in similar work (e.g. Ettori et al. 2003; Allen et al. 2008), we measure a decrease in $\Omega_{\mathrm{m}}$ by about 9 per cent (see case labelled " $f_{\text {star,W"). }}$

To select our sample of 57 clusters, we have applied only the selection criterion of $T_{\text {gas }}>4 \mathrm{keV}$ to consider only the most massive systems for which the ICM physics should be mostly determined by the process of gravitational collapse.

Once we add the further criterion that the relative uncertainty on the gas mass fraction values has to be lower than 0.5 $\left(\epsilon_{\text {gas }} / f_{\text {gas }}<0.5\right)$, the sample is reduced to 41 objects and the good fit obtained $\left(\chi^{2}=34.6\right)$ provides marginally looser constraints on the cosmological parameters, but in better agreement with WMAP 5-year limits. On the other hand, when very hot systems $\left(T_{\text {gas }}>8 \mathrm{keV}\right)$ are considered, the sample is reduced to only 22 objects and estimates of $\Omega_{\Lambda}$ consistent with zero are obtained. Similar constraints are obtained when the local $(z<0.3)$
8 clusters are excluded due to the insensitivity of the geometrical part of the method to the dark energy component at high redshift.

In our selection, we do not consider the morphological aspect of the cluster. To study this effect, we have considered the measurements of the centroid shift for the 43 objects in common with the sample of 90 clusters analysed by Maughan et al. (2007) with $T_{\text {gas }}>4 \mathrm{keV}$. We consider as relaxed objects the 18 clusters with a centroid shift lower than the median value of $1.18 \times 10^{-2}$ measured over the entire sample of 115 clusters (see their Table 2). These 18 objects are part of the first sample, labelled $c e n_{0}$. A second sample, $c e n_{1}$, includes the 16 objects for which an estimate of the centroid shift is not available, for a total number of 34 clusters. Through these two samples, we obtain very similar results, with a relativly larger (smaller) contributions from the dark energy (matter) component compared to the reference values.

Overall, we can attribute a systematic relative error of about \pm 5 per cent to the most robust cosmological constraint, $\Omega_{\mathrm{m}}$. It is estimated by considering the standard deviations of the values plotted in Fig. 11 and summarized in Table 3 around our best-fit reference value of $\Omega_{\mathrm{m}}=0.35$. Similarly, we associate a systematic uncertainty of $(-0.25,+0.30)$ to our best-fit value $\Omega_{\Lambda}=0.59$ (about 45 per cent) and a relative systematic uncertainty of $(-25,+34)$ per cent to our best-fit value of $w=-1.1$.

\subsection{The reverse situation: the baryon distribution in X-ray galaxy clusters after WMAP 5-year}

In the previous sections, we have looked at the best-fit cosmological parameters that better reproduce the observed distribution of the cluster baryonic mass fraction. We now reverse the the problem, by fixing the cosmological parameters to the values suggested from the analysis of the anistropies in the cosmic microwave background and investigating the average physical properties of the baryons in our X-ray luminous galaxy clusters.

Given the above measurements of the cluster gas and total masses, and the estimated contribution from the stellar component, we can estimate the relative distribution of baryons in galaxy clusters assuming that the cosmology is defined in its components from best-fit results obtained after the WMAP 5-year release (Dunkley et al. 2008), combined in a joint analysis with supernovae type Ia data and Baryon Acoustic Oscillations constraints (see Komatsu et al. 2008). In the present work, we follow the analysis described by Ettori (2003) and update the results discussed there.

We estimate the relative contribution of the cluster baryons to the cosmic value $\left(\Omega_{\mathrm{b}} / \Omega_{\mathrm{m}}\right)_{\mathrm{WMAP}}=0.165 \pm 0.009$, where the best-fit results after the WMAP 5-year release are considered $\left(H_{0}=70.1 \pm 1.3 \mathrm{~km} \mathrm{~s}^{-1} \mathrm{Mpc}^{-1}, \Omega_{\mathrm{m}}=1-\Omega_{\Lambda}=0.279 \pm 0.013\right.$, $\Omega_{\mathrm{b}}=0.0462 \pm 0.0015$; see Table 1 in Komatsu et al. 2008). We correct our baryon fractions by the depletion parameter with consistent results both when we use $b=$ constant and $b=b(z)$. In the panel on the left of Fig. 12, we show the mean values (after 1000 bootstrap resamplings) of the ratios between the mass fractions in hot / cold / the remaining baryons and the cosmic value equals to $b \times\left(\Omega_{\mathrm{b}} / \Omega_{\mathrm{m}}\right)_{\mathrm{WMAP}}$.

The requirement for the presence of other baryons apart from the hot, X-ray emitting component and the cold, stellar phase can be explained by either: (i) an underestimate by a factor of 3 of the mass fraction in the form of cold baryons; or (ii) an underestimate by about 20 per cent of $f_{\text {gas }}$.

Recently, McCarthy et al. (2007) obtained similar results by combining $f_{\text {gas }}$ profiles collected from the literature, and corrected for (i) stellar mass contributions; (ii) violations of the 
Table 3. Best-fit results and $1 \sigma\left(\Delta \chi^{2}=1\right)$ errors of the cosmological parameters constrained in our analysis under different assumptions.

\begin{tabular}{|c|c|c|c|c|c|}
\hline & $\Omega_{\mathrm{m}}$ & $\Omega_{\Lambda}$ & $\Omega_{\mathrm{m}}$ & $-w$ & Comments \\
\hline & \multicolumn{2}{|c|}{$w=-1$} & \multicolumn{2}{|c|}{$\Omega_{\mathrm{k}}=0$} & \\
\hline REF & $0.35_{-0.04}^{+0.03}$ & $0.59_{-0.56}^{+0.44}$ & $0.32_{-0.05}^{+0.04}$ & $1.10_{-0.45}^{+0.60}$ & reference case (Sects. 5.1 and 5.2) \\
\hline$T=T_{0}$ & $0.40_{-0.02}^{+0.03}$ & $0.01_{-0.01}^{+0.46}$ & $0.29_{-0.05}^{+0.08}$ & $1.90_{-1.10}^{+2.50}$ & isothermal ICM (Sect. 3) \\
\hline$b=b_{z}$ & $0.36_{-0.04}^{+0.05}$ & $0.65_{-0.50}^{+0.48}$ & $0.34_{-0.05}^{+0.04}$ & $1.20_{-0.45}^{+0.65}$ & depletion parameter dependent on $z$ (Sect. 4.2) \\
\hline$f_{\mathrm{ICL}}, 0.2$ & $0.34_{-0.04}^{+0.03}$ & $0.59_{-0.56}^{+0.40}$ & $0.31_{-0.04}^{+0.04}$ & $1.05_{-0.40}^{+0.60}$ & adding $20 \%$ to $f_{\text {cold }}$ (Sect. 4.1$)$ \\
\hline$f_{\text {star, } \mathrm{W}}$ & $0.32_{-0.03}^{+0.03}$ & $0.49_{-0.48}^{+0.30}$ & $0.30_{-0.04}^{+0.03}$ & $0.95_{-0.35}^{+0.50}$ & $f_{\text {star }}$ from White et al. (1993; Sect. 4.1) \\
\hline$f_{\text {star, } \mathrm{L}}$ & $0.34_{-0.02}^{+0.02}$ & $0.01_{-0.01}^{+0.34}$ & $0.35_{-0.04}^{+0.04}$ & $0.35_{-0.35}^{+0.55}$ & $f_{\text {star }}$ from Lin et al. (2003; Sect. 4.1) \\
\hline$z>0.3$ & $0.36_{-0.08}^{+0.02}$ & $0.19_{-0.18}^{+1.12}$ & $0.26_{-0.09}^{+0.10}$ & $1.35_{-0.75}^{+1.25}$ & only high- $z$ objects \\
\hline$T>8 \mathrm{keV}$ & $0.34_{-0.05}^{+0.03}$ & $0.07_{-0.06}^{+0.18}$ & $0.31_{-0.09}^{+0.09}$ & $0.75_{-0.60}^{+0.85}$ & only hot objects \\
\hline$\epsilon_{f}<0.5$ & $0.34_{-0.03}^{+0.02}$ & $0.01_{-0.01}^{+0.64}$ & $0.33_{-0.04}^{+0.04}$ & $0.70_{-0.45}^{+0.45}$ & only measurements with lower uncertainty \\
\hline $\mathrm{cen}_{0}$ & $0.29_{-0.05}^{+0.08}$ & $1.35_{-0.52}^{+0.20}$ & $0.24_{-0.05}^{+0.08}$ & $2.00_{-1.15}^{+2.45}$ & more relaxed objects (Sect. 5.4) \\
\hline $\operatorname{cen}_{1}$ & $0.32_{-0.04}^{+0.04}$ & $0.89_{-0.64}^{+0.36}$ & $0.31_{-0.05}^{+0.05}$ & $1.10_{-0.50}^{+0.13}$ & extended "cen 0 " sample (Sect. 5.4) \\
\hline$H_{0}=62$ & $0.37_{-0.04}^{+0.04}$ & $0.63_{-0.48}^{+0.64}$ & $0.35_{-0.05}^{+0.03}$ & $1.15_{-0.40}^{+0.50}$ & $H_{0}$ from Sandage et al. (2006) \\
\hline WMAP5 & $0.32_{-0.02}^{+0.03}$ & $1.01_{-0.28}^{+0.28}$ & $0.33_{-0.02}^{+0.02}$ & $1.25_{-0.25}^{+0.30}$ & $H_{0}$ and $\Omega_{\mathrm{b}}$ from Komatsu et al. (2008) \\
\hline
\end{tabular}

The quoted constraints are obtained for each parameter after marginalization. In Fig. 11, the same constraints are plotted and the number of clusters in the sample and the total $\chi^{2}$ are indicated. The single samples are discussed in Sects. 5.1, 5.2 and 5.4.
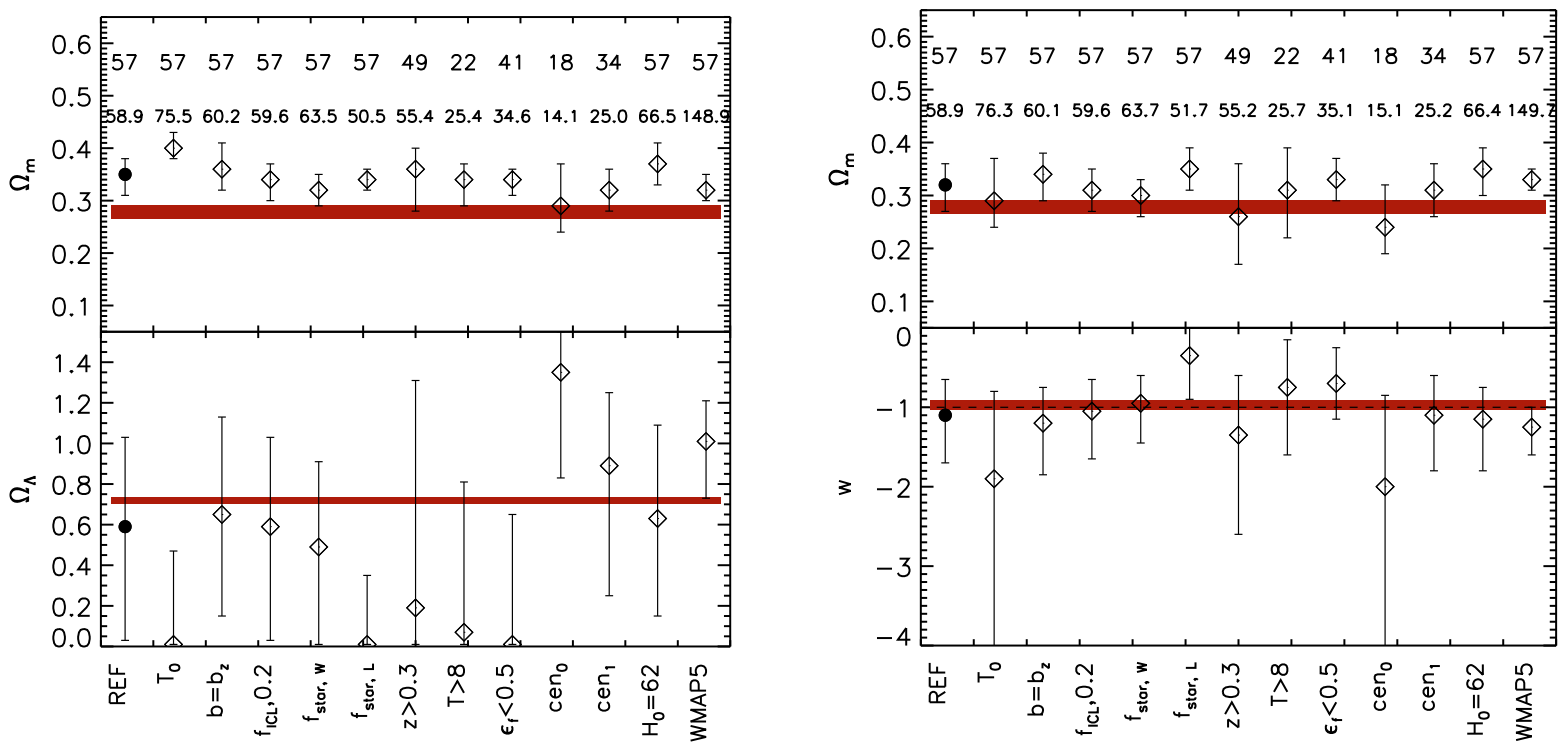

Fig. 11. Best-fit results on $\Omega_{\mathrm{m}}$ and $\Omega_{\Lambda}$ (left) and $\Omega_{\mathrm{m}}$ and $w$ (right) for the different sources of systematics discussed in the text. The shaded regions show the constraints from WMAP 5-year results (Komatsu et al. 2008). All the errors are at the $1 \sigma$ level. The number of clusters that meets the selection criteria and the total $\chi^{2}$ are indicated in the top panel.

hydrostatic equilibrium equation; and (iii) the depletion parameter, with the WMAP 3-year results (Spergel et al. 2007), concluding that the most likely explanation is that $\Omega_{\mathrm{m}}$ must lie in the range $0.28-0.47$, i.e. up to 70 per cent larger than the best-fit from the WMAP 5-year analysis.

We emphasize, however, that our conclusion on a no null contribution from other baryons not accounted for in X-ray emitting plasma and cold stars and intracluster light is drawn mainly from fixing the cosmological parameters to the results of the analysis after the WMAP 5-year release that strongly support a flat Universe solution. On the other hand, our best-fit results seem to suggest either a higher $\Omega_{\mathrm{m}}$ value or, if $\Omega_{\mathrm{m}}$ from the WMAP 5-year constraints is adopted, a higher (lower) $\Omega_{\Lambda}(w)$ value, lowering the requested amount of $f_{\mathrm{ob}}$ (see panel on the right in Fig. 12).

Moreover, by assuming that the WMAP 5-year data provide the "correct" cosmology and, thus, fixing $\left(H_{0}, \Omega_{\mathrm{m}}=1-\Omega_{\Lambda}\right)$ to those values, we can attempt to limit the contribution of other effects that can further bias the estimates of the gas mass fraction. Among these, we consider the level of the ICM clumpiness (e.g. Mathiesen et al. 1999) and the presence of non-thermal pressure support (e.g. Dolag \& Schindler 2000). To weigh their contribution, we look for a minimum $\chi^{2}$ in the parameter space $\{\alpha, C\}$, where $\alpha=P_{\text {Non-thermal }} / P_{\text {thermal }}$ is the ratio, assumed constant in time and space, between a pressure of non-thermal origin and the pressure of the ICM and $C=\left(\left\langle n_{\text {gas }}^{2}\right\rangle /\left\langle n_{\text {gas }}\right\rangle^{2}\right)^{0.5}$ is a measure of the clumpiness of the ICM which is observed in brightness as an average value of the square of the gas density $\left\langle n_{\text {gas }}^{2}\right\rangle$. These two parameters affect the gas mass fraction estimates through propagation of corrections on the total and gas masses in a degenerative way: $M_{\text {tot,new }}=(1+\alpha) M_{\text {tot,old }}$, $M_{\text {gas,new }}=M_{\text {gas,old }} / C$ and, thus, $f_{\text {gas,new }}=f_{\text {gas,old }} /[C(1+\alpha)]$. The net effect is thus to further reduce the gas mass fraction value. Adding this to the indication that the WMAP 5-year cosmology does not account for the observed baryon census will further increase the measured discrepancy. Indeed, we obtain that, at a $3 \sigma$ 

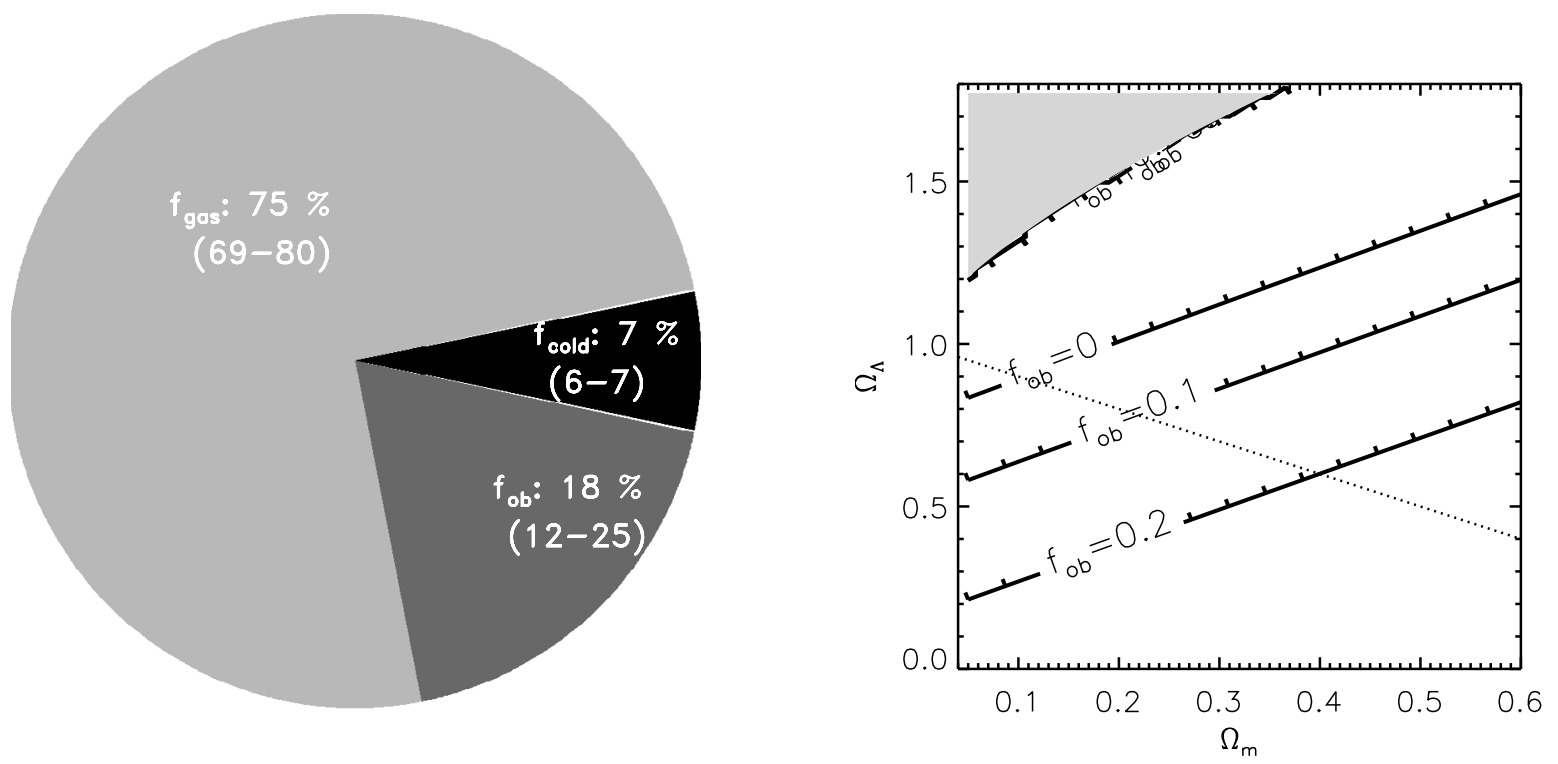

Fig. 12. (Left) Cluster baryonic pie obtained by fixing the cosmological parameters to the best-fit results after the WMAP 5-year release (Komatsu et al. 2008) and considering the mean values of the hot $\left(f_{\mathrm{gas}}\right)$, cold $\left(f_{\text {cold }}\right)$ and other $\left(f_{\mathrm{ob}}\right)$ baryon fractions normalized to the cosmic value, $b\left(\Omega_{\mathrm{b}} / \Omega_{\mathrm{m}}\right)_{\text {WMAP. }}$. Ranges within two standard deviations (95.4\% level of confidence) from the mean (obtained after 1000 bootstrap resamplings) are indicated within the parentheses. (Right) Dependence of the average $f_{\mathrm{ob}}$ value upon the cosmological parameters $\left(\Omega_{\mathrm{m}}, \Omega_{\Lambda}\right)$. The dotted line indicates a flat Universe.

level of confidence with 2 degrees of freedom, our gas mass fraction measurements within $R_{500}$ in a WMAP 5-year concordant Universe constrain $\alpha<0.13$ and $C<1.01$ (the best-fit results require $\alpha=0$ and $C=1$ ).

\section{Conclusions}

We study the baryonic content in 52 X-ray luminous galaxy clusters observed with Chandra in the redshift range 0.3-1.273. We include in the sample the measurements of 8 objects with $k T_{\text {gas }}>4 \mathrm{keV}$ lying in the redshift range $0.06-0.23$ presented in Vikhlinin et al. (2006). We adopt the same functional form to estimate the gas density and temperature profiles to recover the gas and total mass radial distribution. We estimate statistically the average contribution from cold baryons in stars and intracluster light to the total baryonic budget of each cluster. By using the baryonic content determined in this way, we have investigated the robustness of the cosmological constraints provided from the cluster baryon fraction alone.

We show that the a-priori knowledge of the cosmic baryon density $\Omega_{\mathrm{b}}$ and the Hubble constant $H_{0}$ are essential to place significant limits in the $\Omega_{\mathrm{m}}-\Omega_{\Lambda} / w$ plane. In particular, the determination of their central values are required to avoid the introduction of systematic differences in the estimate of the cosmological parameters. This systematic bias cannot be corrected by simply enlarging the error bars on the assumed central values, but need a definitive input from other sources (for instance, CMB measurements, type Ia supernovae data or primordial nucleosynthesis calculations; see, e.g., Allen et al. 2008).

We find that the gas mass fraction measured in our subsample of 57 hot $\left(T_{\text {gas }}>4 \mathrm{keV}\right)$ galaxy clusters, in combination with a Hubble's constant value of $H_{0}=72 \pm 8 \mathrm{~km} \mathrm{~s}^{-1} \mathrm{Mpc}^{-1}$ and $\Omega_{\mathrm{b}} h_{70}^{2}=0.0462 \pm 0.0012$, provides a best-fit result of $\Omega_{\mathrm{m}}=0.35_{-0.04}^{+0.03}$ and $\Omega_{\Lambda}=0.59_{-0.56}^{+0.44}(1 \sigma$ error; $w=-1)$ and $\Omega_{\mathrm{m}}=0.32_{-0.05}^{+0.04}$ and $w=-1.1_{-0.5}^{+0.6}$, when a flat Universe is considered.
We discuss in detail the systematic effect on our best-fit constraints originating from: (i) the stellar mass fraction component, expected to be of the order of $0.1-0.2 f_{\text {gas }}$; (ii) the redshift dependence of the depletion parameter $b$; (iii) the isothermality of the ICM. The latter effect, in particular, increases the total $\chi^{2}$ by 17 and shifts the best-fit values by about 15 percent, pointing out the necessity for a robust and reliable determination of the temperature profiles in galaxy clusters, possibly out to the region where the gas mass fraction measurements are more representative of the cluster baryon content $\left(r \gtrsim R_{2500} \approx 0.3 R_{200}\right)$.

By fixing the cosmological parameters to the values from the WMAP 5-year data analysis, we limit the contributions expected from non-thermal pressure support and ICM clumpiness to be lower than about 10 per cent. Otherwise, there is room to accommodate baryons not accounted for either in the X-ray emitting plasma or in stars in the order of 18 per cent of the total cluster baryon budget. However, this value is lowered to zero once a no-flat Universe is allowed, as suggested from the cluster gas mass fraction distribution alone.

Bearing in mind that the $f_{\text {gas }}$ method to constrain the cosmological parameters is not self-consistent and needs a strong apriori knowledge of both the Hubble constant value and the cosmic baryon density, we conclude that it offers well proven and reliable results on $\Omega_{\mathrm{m}}$ (relative statistical error at $1 \sigma$ of $\sim 11 \%$, with a further systematic effect of $\pm 5 \%$ ), whereas it presents weakness in terms of the assumptions on the astrophysics involved when applied to investigate the dark energy issue. On the other hand, this method provides a complementary and independent constraint of the parameter space investigated from planned dark energy experiments (e.g. Rapetti et al. 2008; see also Linder 2007 for a more pessimistic view) and should be supported for this application.

Acknowledgements. We acknowledge the financial contribution from contracts ASI-INAF I/023/05/0 and I/088/06/0. PT and SB acknowledge the financial support from the PD51 INFN grant. Cristiano De Boni is thanked for the useful discussion. 


\section{References}

Allen, S. W., Schmidt, R. W., \& Fabian, A. C. 2001, MNRAS, 328, L37 Allen, S. W., Schmidt, R. W., \& Fabian, A. C. 2002, MNRAS, 334, L11 Allen, S. W., Rapetti, D. A., Schmidt, R. W., et al. 2008, MNRAS, 383, 879 Anders, E., \& Grevesse, N. 1989, Geochim. Cosmochim. Acta, 53, 197 Arnaud, K. A. 1996, Astronomical Data Analysis Software and Systems V, ed. G. Jacoby, \& J. Barnes, ASP Conf. Ser., 101, 17

Arnaud, M., Rothenflug, R., Boulade, O., Vigroux, L., \& Vangioni-Flam, E. 1992, A\&A, 254, 49

Balestra, I., Tozzi, P., Ettori, S., et al. 2007, A\&A, 462, 429

Caldwell, R. R., Dave, R., \& Steinhardt, P. J. 1998, Phys. Rev. Lett., 80, 1582

Carroll, S. M., Press, W. H., \& Turner, E. L. 1992, ARA\&A, 30, 499

Castillo-Morales, A., \& Schindler, S. 2003, A\&A, 403, 433

David, L. P., Jones, C., \& Forman, W. 1995, ApJ, 445, 578

Dolag, K., \& Schindler, S. 2000, A\&A, 364, 491

Dunkley J., et al. 2008, ApJS, in press [arXiv: 0803.0586] (WMAP 5-year)

Ettori, S. 2000, MNRAS, 311, 313

Ettori, S. 2001, MNRAS, 323, L1

Ettori, S., \& Fabian, A. C. 1999a, MNRAS, 305, 834

Ettori, S., \& Fabian, A. C. 1999b, proceedings of the Ringberg workshop on Diffuse Thermal and Relativistic Plasma in Galaxy Clusters, ed. H. Böhringer,

L. Feretti, \& P. Schuecker, MPE Report No. 271, MPE Garching

Ettori, S., \& Brighenti, F. 2008, MNRAS, 387, 631

Ettori, S., Tozzi, P., \& Rosati, P. 2003, A\&A, 398, 879

Ettori, S., Dolag, K., Borgani, S., \& Murante, G. 2006, MNRAS, 365, 1021

Evrard, A. E. 1997, MNRAS 292, 289

Freedman, W. L., Madore, B. F., Gibson, B. K., et al. 2001, ApJ, 553, 47

Frenk, C. S., White, S. D. M., Bode, P., et al. 1999, ApJ, 525, 554

Fukugita, M., Hogan, C. J., \& Peebles, P. J. E. 1998, ApJ, 503, 518

Gonzalez, A. H., Zaritsky, D., \& Zabludoff, A. I. 2007, ApJ, 666, 147

Hannestad, S., \& Raffelt, G. G. 2006, JCAP, 11, 16

Komatsu, E. et al. 2008, ApJS, in press [arXiv: 0803 . 0547] (WMAP 5-year)

Kravtsov, A. V., Nagai, D., \& Vikhlinin, A. 2005, ApJ, 625, 588

Lagana, T. F., Lima Neto, G. B., Andrade-Santos, F., \& Cypriano, E. S. 2008, A\&A, 485, 633
Lin, Y.-T., \& Mohr, J. J. 2004, ApJ, 617, 879

Lin, Y.-T., Mohr, J. J., \& Stanford, S. A. 2003, ApJ, 591, L749

Linder, E. V. 2007, JCAP, 04, 4

McCarthy, I. G., Bower, R. G., \& Balogh, M. L. 2007, MNRAS, 377, 1457

Markevitch, M., \& Vikhlinin, A. 2001, ApJ, 563, 95

Mathiesen, B., Evrard, A. E., \& Mohr, J. J. 1999, ApJ, 520, L21

Maughan, B. J., Jones, C., Jones, L. R., \& Van Speybroeck, L. 2007, ApJ, 659, 1125

Murante, G., Arnaboldi, M., Gerhard, O., et al. 2004, ApJ, 607, L83

Murante, G., Giovalli, M., Gerhard, O., et al. 2007, MNRAS, 377, 2

Mohr, J. J., Mathiesen, B., \& Evrard, A. E. 1999, ApJ, 517, 627

Navarro, J. F., Frenk, C. S., \& White, S. D. M. 1997, ApJ, 490, 493

Peebles, P. J. E., \& Ratra, B. 2003, Rev. Mod. Phys., 75, 559

Rapetti, D., Allen, S. W., \& Weller, J. 2005, MNRAS, 360, 555

Rapetti, D., Allen, S. W., \& Mantz, A. 2008, MNRAS, 388, 1265

Rines, K., Forman, W., Pen, U., Jones, C., \& Burg, R. 1999, ApJ, 517, 70

Rosati, P., Borgani, S., \& Norman, C. 2002, ARA\&A, 40, 539

Roussel, H., Sadat, R., \& Blanchard, A. 2000, A\&A, 361, 429

Sadat, R., Blanchard, A., Vauclair, S. C., et al. 2005, A\&A, 437, 31

Sandage, A., Tammann, G. A., Saha, A., et al. 2006, ApJ, 653, 843

Santos, J. S., Rosati, P., Tozzi, P., et al. 2008, A\&A, 483, 35

Sasaki, S. 1996, PASJ, 48, L119

Spergel, D. N., Verde, L., Peiris, H. V., et al. 2003, ApJS, 148, 175 (WMAP 1-year)

Spergel, D. N., Bean, R., Doré, O., et al. 2007, ApJS, 170, 377 (WMAP 3-year)

Stanford, S. A., Holden, B. P., Rosati, P., et al. 2001, ApJ, 552, 504

Steigman, G. 2006, Int. J. Mod. Phys., E15, 1

Strazzullo, V., Rosati, P., Stanford, S. A., et al. 2006, A\&A, 450, 909

Turner, M. S., \& White, M. 1997, Phys. Rev. D, 56 (8), 4439

Vikhlinin, A., Kravtsov, A., Forman, W., et al. 2006, ApJ, 640, 691

Voevodkin, A., \& Vikhlinin, A. 2004, ApJ, 601, 610

Wang, L., \& Steinhardt, P. J. 1998, ApJ, 508, 483

White, D. A., \& Fabian, A. C. 1995, MNRAS, 273, 72

White, S. D. M., Navarro, J. F., Evrard, A. E., et al. 1993, Nature, 366, 429 
S. Ettori et al.: Cosmological constraints from the cluster gas fraction, Online Material $p 1$

\section{Appendix A: Fit of the deprojected gas density profiles}

We show the electron density profiles, obtained from the deprojection of the surface brightness, of the objects in our high- $z$ sample. We overplot the best-fitted function form in Eq. (5) fitted over the radial range $0-R_{\text {spat }}$. Both the best-fit parameters and $R_{\text {spat }}$ are quoted in Table 1.
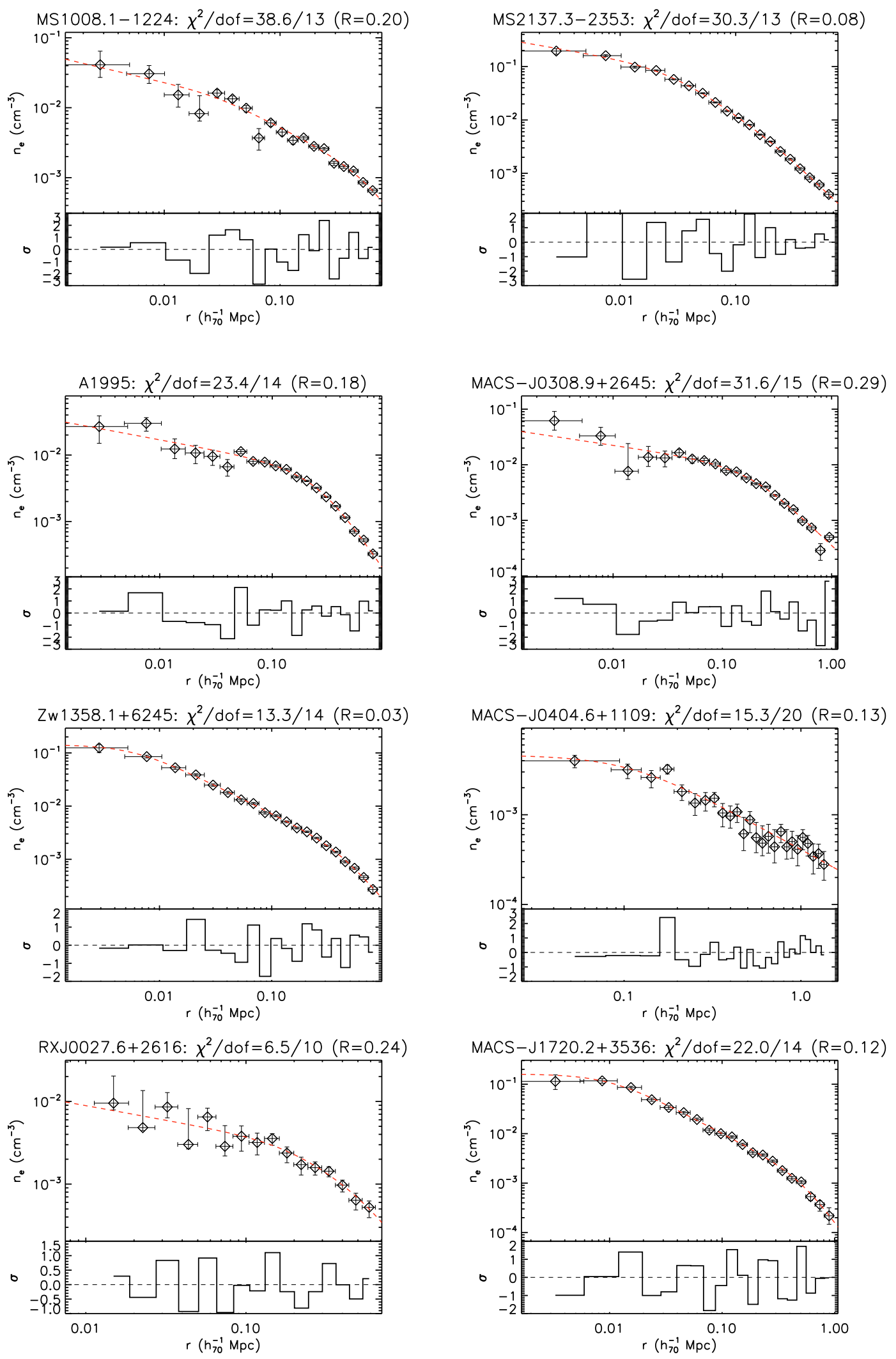

Fig. A.1. Plots of the deprojected electron density profiles with the best-fit model in Eq. (5) overplotted and the residuals $\sigma_{i}=\left(d_{i}-m_{i}\right) / \epsilon_{i}$ shown in the bottom panel, where $d_{i}, m_{i}$ and $\epsilon_{i}$ are the values of the data, model and error on the data at a given radius, respectively. Together with the $\chi^{2}=\sum\left(d_{i}-m_{i}\right)^{2} / \epsilon_{i}^{2}=\sum \sigma_{i}^{2}$ value, the $R$ factor represents a measure of the overall residuals and is given by $R=\sum$ abs $\left(d_{i}-m_{i}\right) / \sum d_{i}$. In 81 per cent of the cases, $R<0.2$. 
S. Ettori et al.: Cosmological constraints from the cluster gas fraction, Online Material p 2
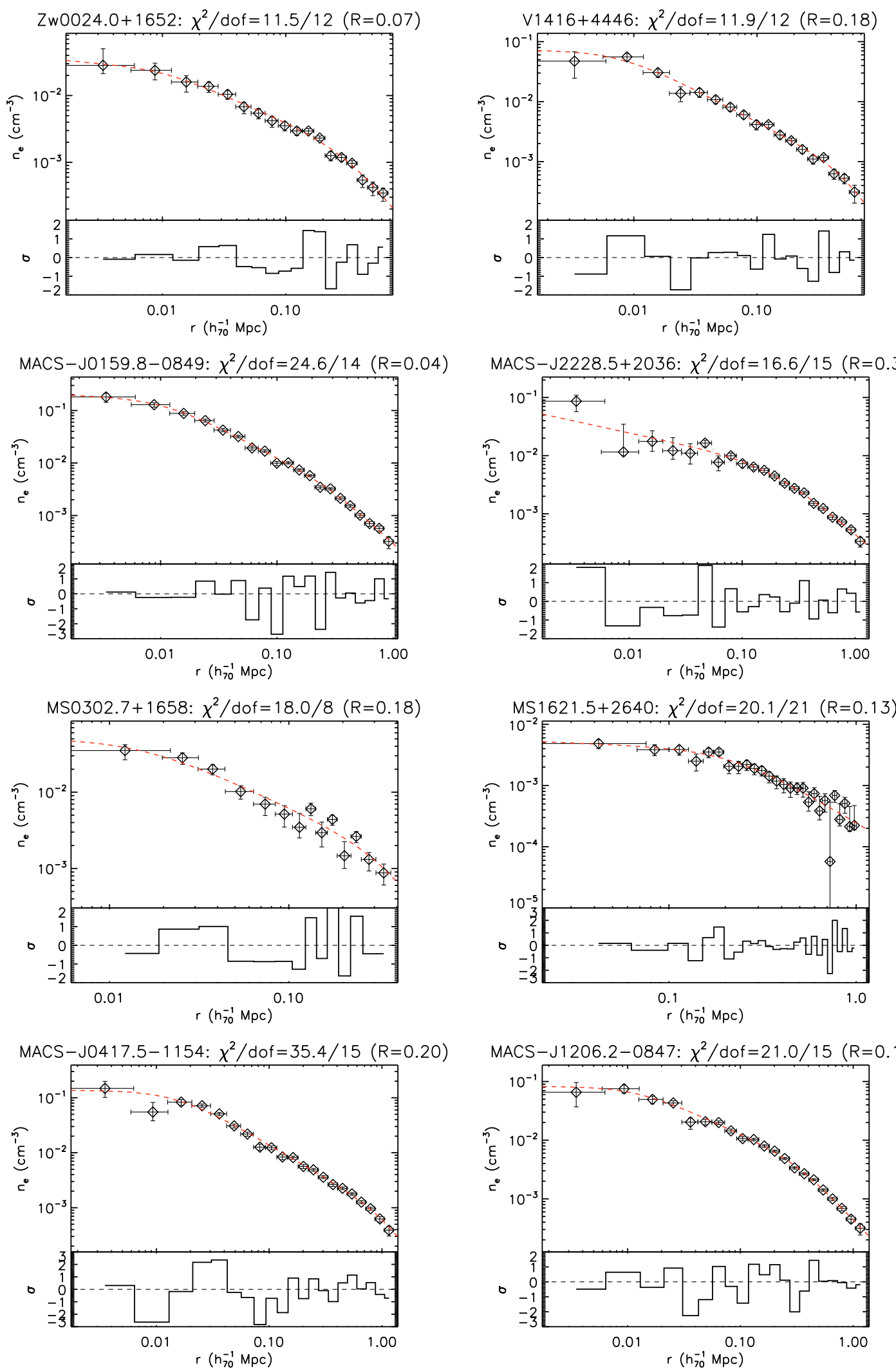

Fig. A.1. continued. 
S. Ettori et al.: Cosmological constraints from the cluster gas fraction, Online Material $p 3$
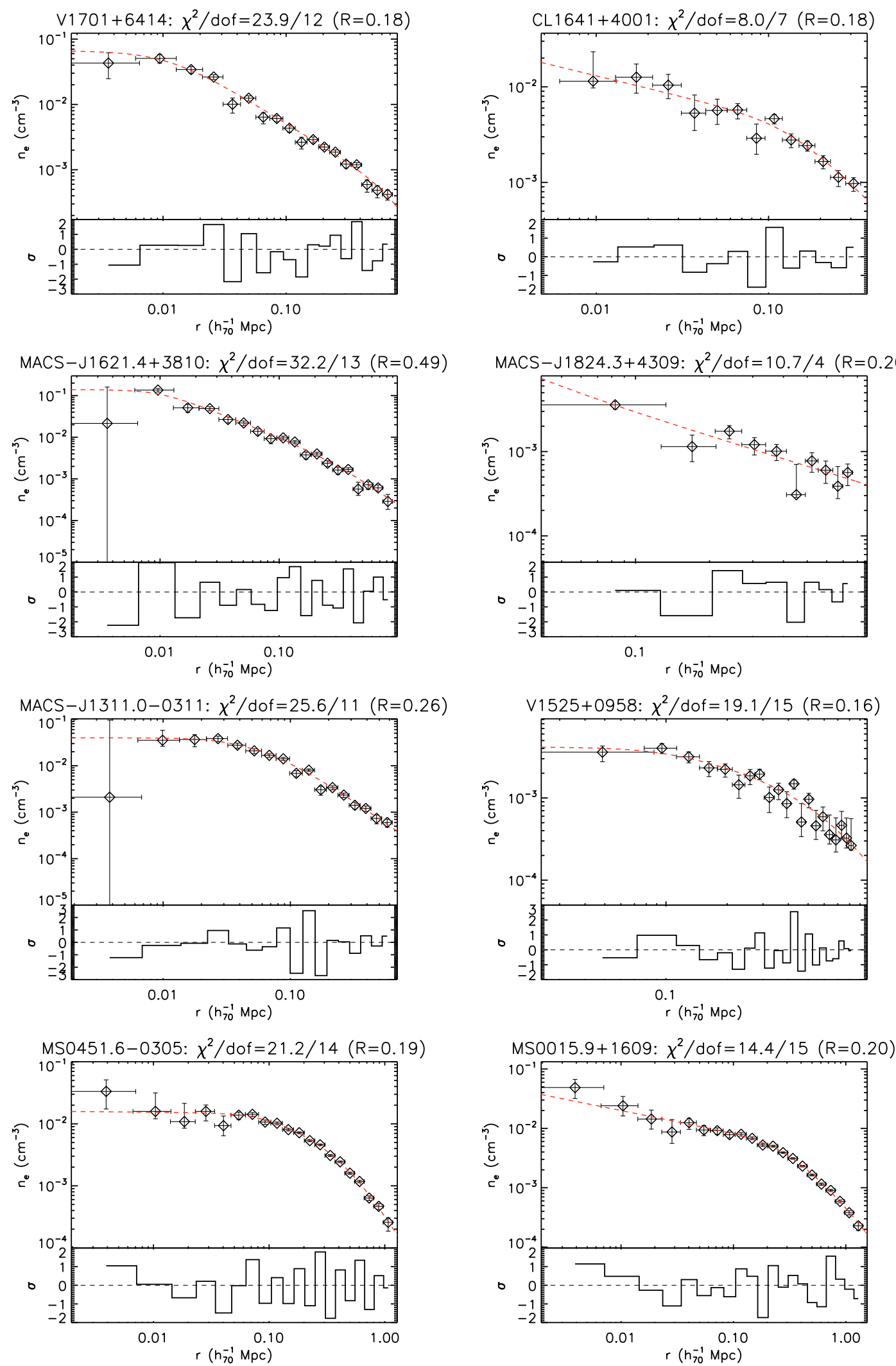

Fig. A.1. continued. 
S. Ettori et al.: Cosmological constraints from the cluster gas fraction, Online Material p 4
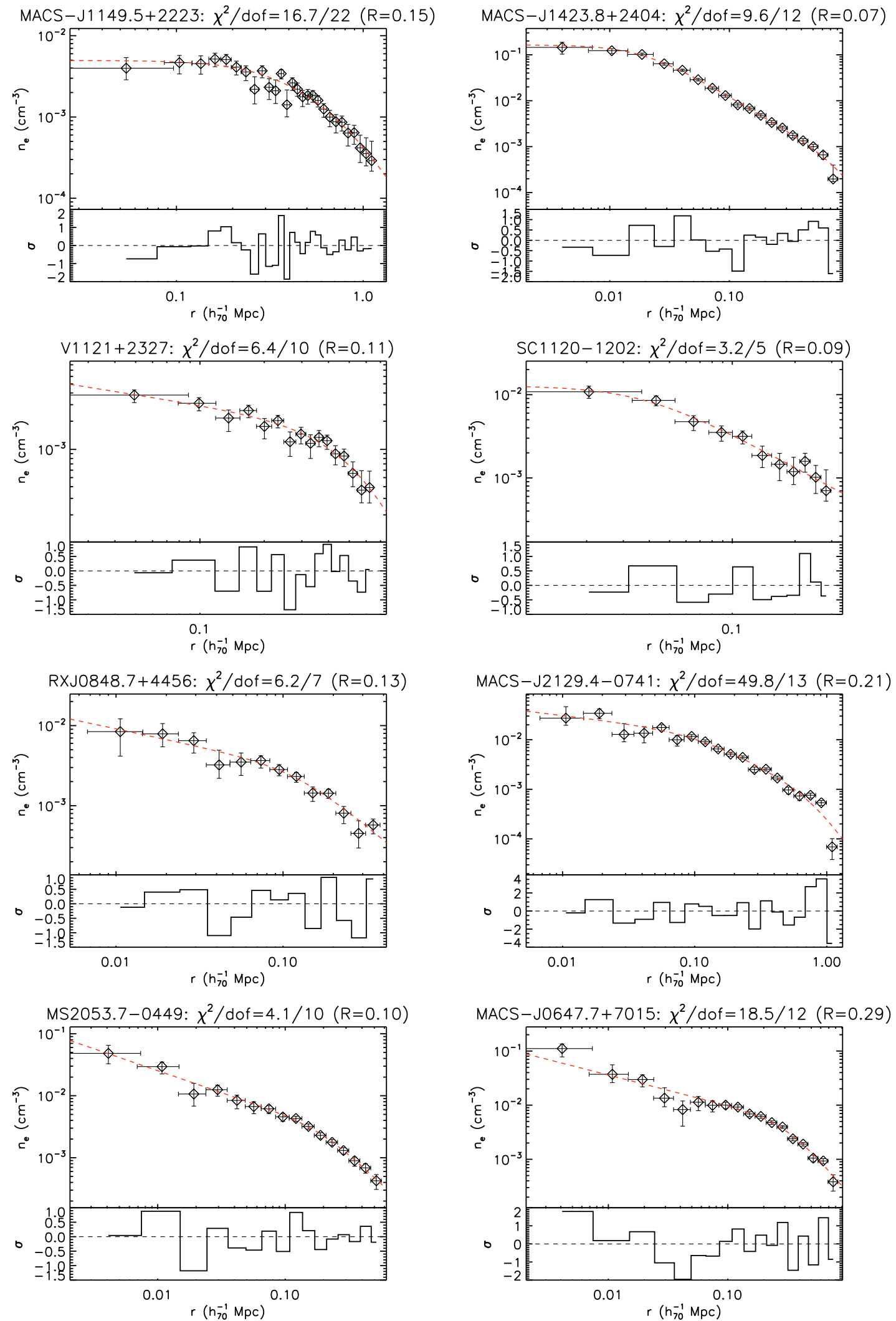

Fig. A.1. continued. 
S. Ettori et al.: Cosmological constraints from the cluster gas fraction, Online Material p 5
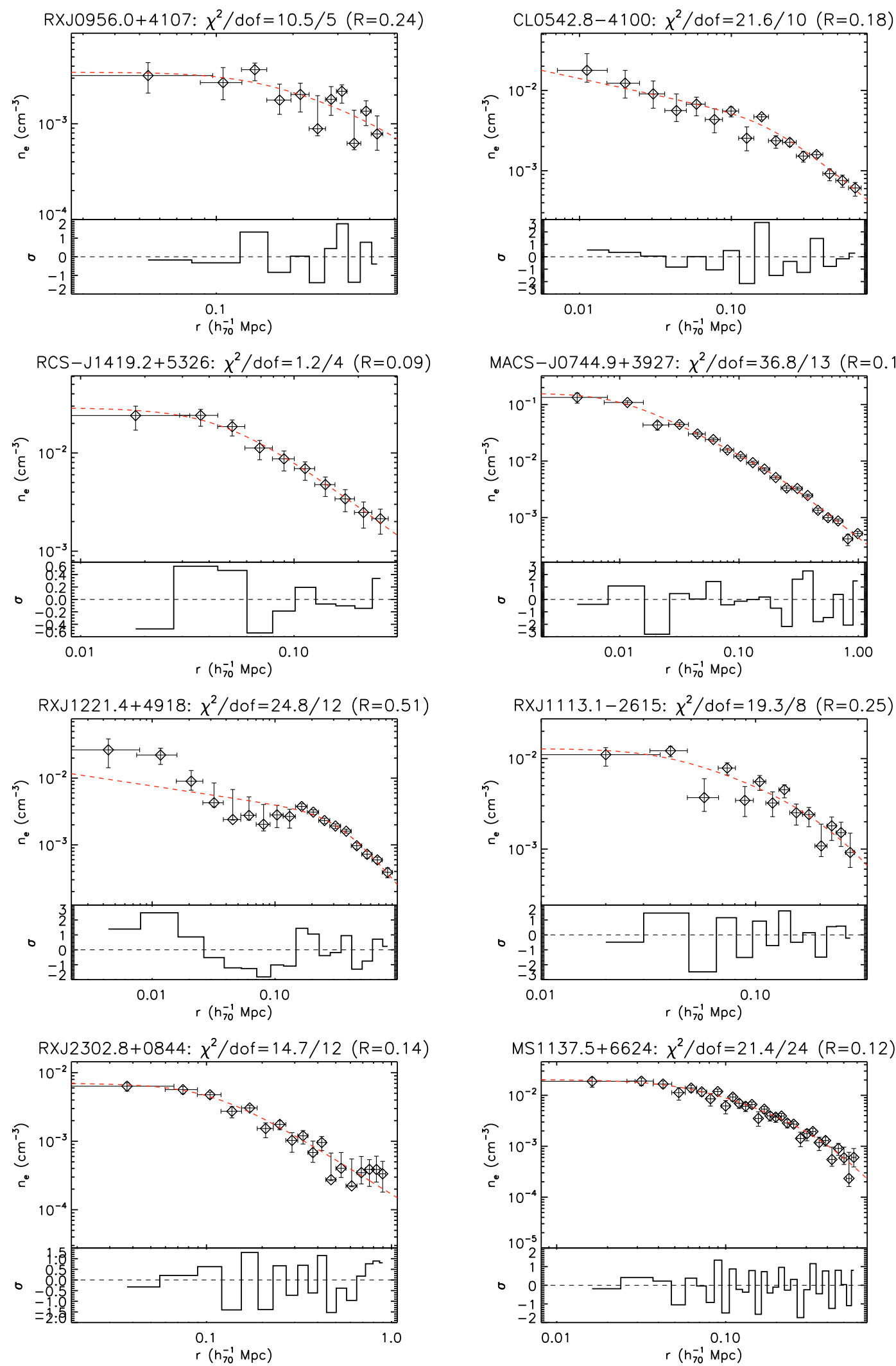

Fig. A.1. continued. 
S. Ettori et al.: Cosmological constraints from the cluster gas fraction, Online Material p 6
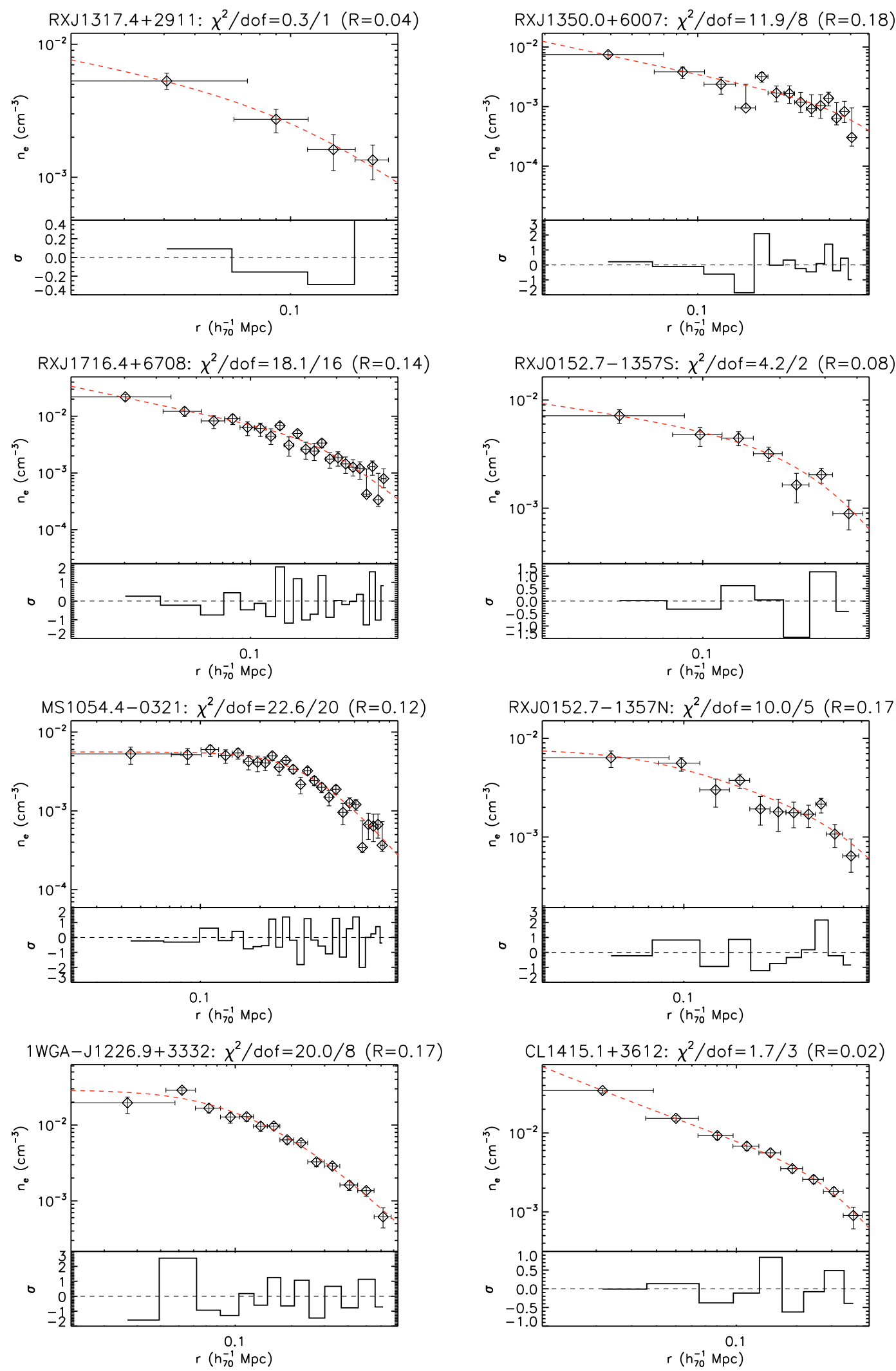

Fig. A.1. continued. 
S. Ettori et al.: Cosmological constraints from the cluster gas fraction, Online Material p 7
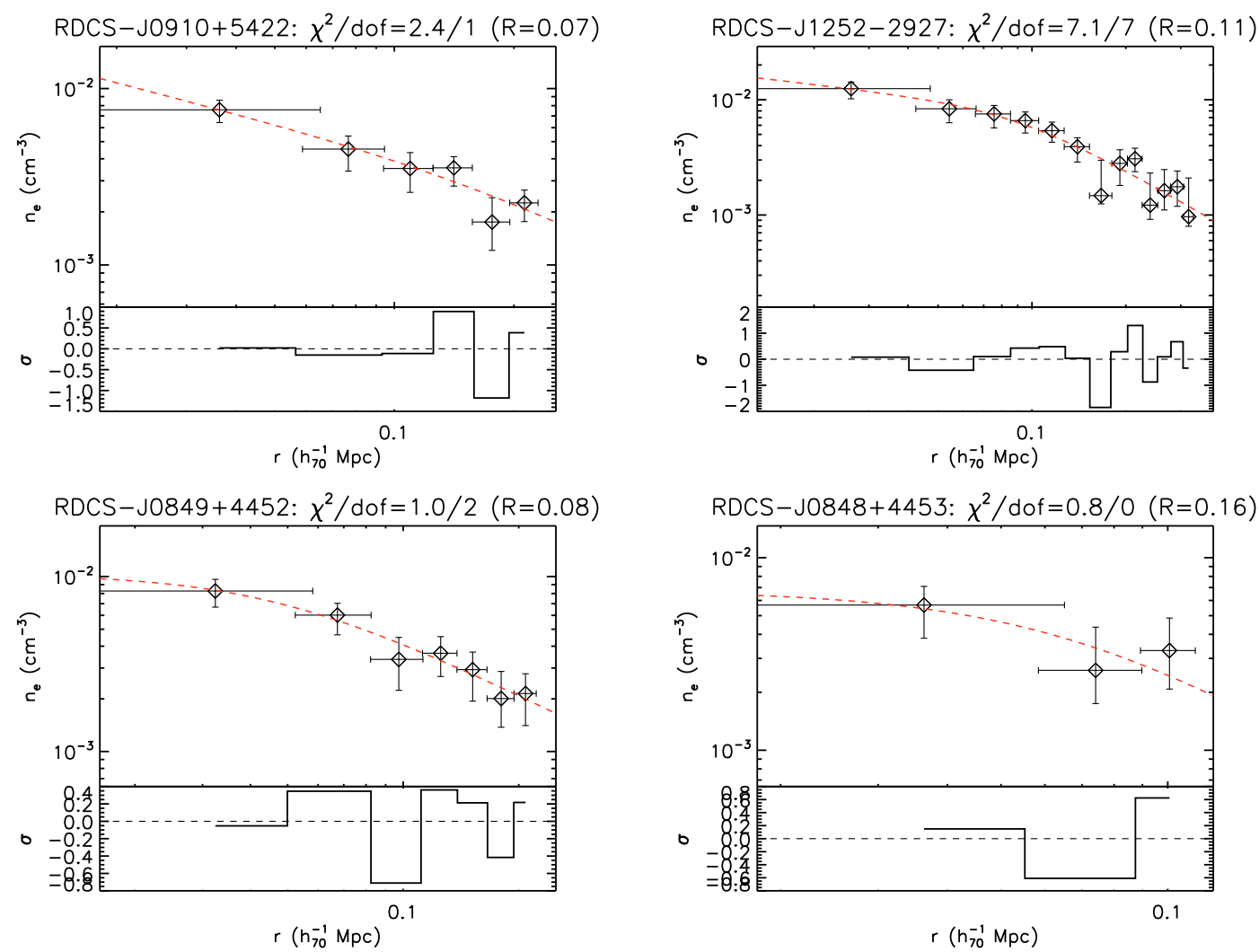

Fig. A.1. continued. 